\title{
MONOIDAL FUNCTORS, ACYCLIC MODELS AND CHAIN OPERADS
}

\author{
F. GUILLÉN SANTOS, V. NAVARRO, P. PASCUAL, AND A. ROIG
}

\begin{abstract}
We prove that for a topological operad $P$ the operad of oriented cubical chains, $C_{*}^{\text {ord }}(P)$, and the operad of singular chains, $S_{*}(P)$, are weakly equivalent. As a consequence, $C_{*}^{\text {ord }}(P ; \mathbb{Q})$ is formal if and only if $S_{*}(P ; \mathbb{Q})$ is formal, thus linking together some formality results that are spread out in the literature. The proof is based on an acyclic models theorem for monoidal functors. We give different variants of the acyclic models theorem and apply the contravariant case to study the cohomology theories for simplicial sets defined by $R$-simplicial differential graded algebras.
\end{abstract}

\section{Contents}

1. Introduction 2

2. $\quad$ The monoidal background 5

$\begin{array}{lll}2.1 . & \text { Monoidal categories } & 5\end{array}$

$\begin{array}{ll}2.2 . & \text { Monoidal functors } \\ \end{array}$

$\begin{array}{ll}2.3 . & 7\end{array}$

$\begin{array}{llr}2.4 . & \text { Comonoidal functors } & 8\end{array}$

\begin{tabular}{lll}
\hline 3. & Shuffle and Alexander-Whitney maps & 8
\end{tabular}

3.1. $\quad$ Simplicial chain complexes 1

3.2. The simple functors 9

\begin{tabular}{lll}
\hline 3.3. & Symmetric simple functor & 10
\end{tabular}

\begin{tabular}{lll}
\hline 4. An acyclic models theorem for monoidal functors & 10
\end{tabular}

\begin{tabular}{lll}
\hline 4.1. Acyclic classes and weak equivalences & 10
\end{tabular}

$\begin{array}{lll}4.2 . & \text { G-presentable objects and G-weak equivalences } & 13\end{array}$

4.3. The acyclic models theorem for monoidal functors 15

\begin{tabular}{lll}
\hline 4.4. & Weak homotopy type & 16
\end{tabular}

$\begin{array}{ll}4.5 . & 16\end{array}$

${ }^{1}$ Partially supported by projects DGCYT BFM2003-60063 and BFM2003-06001

Date: December 21, 2012. 
5. Application: comparison of singular and cubical chains 17

$\begin{array}{ll}5.1 . & 17\end{array}$

$\begin{array}{lll}5.2 . & \text { A monoidal cotriple in Top } & 17\end{array}$

$\begin{array}{ll}5.3 . & 18\end{array}$

\begin{tabular}{lll}
\hline 6. & Symmetric monoidal functors & 19
\end{tabular}

$\begin{array}{lll}6.1 . & \text { Acyclic models for symmetric monoidal functors } & 19\end{array}$

$\begin{array}{lll}6.2 . & \text { The Kleisli cotriple } & 20\end{array}$

\begin{tabular}{lll}
\hline 6.3. & Ordered cubical chains & 20
\end{tabular}

\begin{tabular}{lll}
\hline 7. Application to operads & 22 \\
\hline
\end{tabular}

\begin{tabular}{lll}
\hline 7.1. & Operads & 22
\end{tabular}

$\begin{array}{ll}7.2 . & 23\end{array}$

\begin{tabular}{lll}
\hline 7.3. & Formality & 24
\end{tabular}

\begin{tabular}{lll}
\hline 7.4. & Modular operads & 25
\end{tabular}

8. Contravariant functors 25

$\begin{array}{ll}\text { 8.1. The standard construction } & 26\end{array}$

8.2. Acyclic models for contravariant monoidal functors 26

8.3. Application to singular and cubical cochains 26

\begin{tabular}{lll}
\hline 9. Application to cohomology theories & 27
\end{tabular}

\begin{tabular}{lll}
\hline 9.1. & Cohomology theories & 27 \\
\hline
\end{tabular}

$\begin{array}{ll}9.2 . & 30\end{array}$

$\begin{array}{ll}\text { References } & 31\end{array}$

\section{INTRODUCTION}

Since its introduction by S. Eilenberg and S. MacLane in [EM1, the acyclic models theorem has been a powerful technique in algebraic topology and homological algebra. It says that, if $K_{*}, L_{*}$ : $\mathcal{A} \longrightarrow \mathbf{C}_{*}(\mathbb{Z})$ are functors from a category with models $\mathcal{A}$ to the category of non-negative chain complexes of abelian groups $\mathbf{C}_{*}(\mathbb{Z})$, such that $K_{*}$ is representable and $L_{*}$ is acyclic on models, then any morphism $f_{-1}: H_{0}\left(K_{*}\right) \longrightarrow H_{0}\left(L_{*}\right)$ extends to a morphism $f_{*}: K_{*} \longrightarrow L_{*}$, and this extension is unique up to homotopy. In particular, if both $K_{*}, L_{*}$, are representable and acyclic on models, then any isomorphism $f_{-1}: H_{0}\left(K_{*}\right) \cong H_{0}\left(L_{*}\right)$ extends to a homotopy equivalence $f_{*}: K_{*} \simeq L_{*}$, unique up to homotopy, thus defining an isomorphism $H f_{*}: H\left(K_{*}\right) \longrightarrow H\left(L_{*}\right)$. 
In some elementary presentations, the representability hypothesis for $K_{*}$ is replaced by asking it to be free on models, see for instance [D].

In [EM1, Eilenberg-MacLane apply their theorem to compare singular chains $S_{*}(X)$ and normalized cubical chains $C_{*}(X)$ : for $p \geq 0$, the abelian group $S_{p}(X)$ is freely generated by continuous maps $\Delta^{p} \longrightarrow X$, and for cubical chains one takes first the free group $Q_{p}(X)$ of continuous maps $I^{p} \longrightarrow X$, and defines $C_{p}(X)$ as the quotient of $Q_{p}(X)$ by the degenerated cubical chains (observe that $C_{*}(X)$ is denoted by $Q_{*}^{N}(X)$ in [EM1). While $S_{*}(X)$ is free on models, $C_{*}(X)$ is not. Nevertheless, $C_{*}(X)$ is representable, so acyclic models allows us to extend the identification $S_{0}(X)=C_{0}(X)$ to a natural homotopy equivalence $S_{*}(X) \longrightarrow C_{*}(X)$. In this way, the two complexes define a homology functor $H_{*}(X ; \mathbb{Z})$ from the category Top of topological spaces to the category of graded abelian groups, up to canonical isomorphism.

Moreover, acyclic models permit to define Eilenberg-Zilber equivalences in both theories, which together with the Künneth theorem endow the homology functor with morphisms $H_{*}(X ; \mathbb{Z}) \otimes$ $H_{*}(Y ; \mathbb{Z}) \longrightarrow H_{*}(X \times Y ; \mathbb{Z})$, to which we will refer as Künneth morphisms, which are independent of the theory we use, up to isomorphism. In categorical terms, the Künneth morphisms define a monoidal structure on the functor $H_{*}(; \mathbb{Z})$, so we can say that the monoidal functor of homology is well defined up to isomorphism.

Since the appearance of [EM1], there have been many variations and generalizations of the acyclic models theorem, according to the field of expected applications; see, for instance, GMo], [BB], [DMO], [K1] and [B96], to cite a few. When dealing with products, acyclic models allow us to produce a whole family of higher homotopies from which we can deduce the multiplicativity of morphisms in cohomology. For example, the natural integration morphism $\int: S^{*}(X) \longrightarrow$ $\Omega^{*}(X)$ is not multiplicative, but the higher homotopies defined by acyclic models define a dash morphism which induces a multiplicative morphism between the singular cohomology and the De Rham cohomology of $X$, (see [BG], [Mu]).

For us, the most relevant version of acyclic models is that of M. Barr in [B96], also exposed in his book [B02]. The Eilenberg-MacLane theorem gives morphisms $K_{*} \longrightarrow L_{*}$ well defined up to homotopy, so that in homology they define morphisms up to isomorphism. In [B96], Barr looks for uniquely defined morphisms in a conveniently localized category of complexes. In this way, Barr's version of acyclic models eliminates the indeterminacy up to homotopy fund in the category of complexes remaining at the complex level and, most remarkably, it allows to look for extensions defined by a chain of true morphims $K_{*} \rightarrow \cdot \leftarrow \cdots \rightarrow L_{*}$ in either direction.

Since the functors $S_{*}, C_{*}$ are monoidal functors, they transform non symmetric operads into non symmetric operads, so we have the non symmetric $d g$ operads $S_{*}(P)$ and $C_{*}(P)$ associated to a given topological operad $P$. Although one expects these two $d g$ operads to be homotopy equivalent, the known acyclic models theorems does not apply, because the transformation $S_{*} \longrightarrow C_{*}$ deduced above is not necessarily a monoidal natural transformation of monoidal functors and, consequently, we do not necessarily obtain an operad morphism between $S_{*}(P)$ and $C_{*}(P)$. This situation is reminiscent of what happens with products in the De Rham theorem cited above. 
Our main result, theorem 4.3.1, is an acyclic models theorem for monoidal functors from a monoidal category $\mathcal{C}$ to the monoidal category $\mathbf{C}_{*}(\mathbb{Z})$. We likewise establish several variations of this result, which cover the symmetric monoidal and the contravariant monoidal settings. As a consequence of our results we prove that $S_{*}$ and $C_{*}$ are weakly equivalent as monoidal functors, so it follows that, for a given topological operad $P$, the non symmetric $d g$ operads $S_{*}(P)$ and $C_{*}(P)$ are weakly equivalent. We deduce also a multiplicative De Rham comparison result, without reference to dash morphisms.

Theorem 4.3.1 is stated and proved by adapting to the monoidal setting the categorical framework proposed by Barr in [B96]. Thus, if $\mathcal{C}$ is a monoidal category, $K_{*}, L_{*}$ are monoidal functors from $\mathcal{C}$ to $\mathbf{C}_{*}(\mathbb{Z})$, and $H_{0}\left(K_{*}\right) \longrightarrow H_{0}\left(L_{*}\right)$ is a monoidal morphism, theorem 4.3.1 establishes sufficient conditions on $K_{*}, L_{*}$ to extend $f$ to a morphism $f: K_{*} \longrightarrow L_{*}$ defined up to weak equivalence, that is, a morphism in the localized category obtained by inverting a given class of weak equivalences.

The main tools in the statement and proof of theorem 4.3.1 are the models of $\mathcal{C}$ and the weak equivalences. The models are given by a suitable monoidal cotriple $\mathbf{G}$ defined in $\mathcal{C}$. In the classical situation the cotriple $\mathbf{G}$ is a model-induced cotriple. For instance, in Top one can take $\mathbf{G}(X)=\sqcup \Delta^{n}$, where the disjoint union is taken over all $n \geq 0$ and all continuous maps $\Delta^{n} \longrightarrow X$. In the paper, we use a monoidal version of this cotriple, while when dealing with symmetric monoidal functors from Top to $\mathbf{C}_{*}(\mathbb{Z})$ we use the cotriple introduced by Kleisli in [K2]. As for the class of morphisms $\Sigma$, in general it is associated to an acyclic class of complexes (see [B96]), and in the applications it is a class in between objectwise homotopy equivalences and quasi-isomorphisms.

We apply theorem 4.3.1 and its variations in two different contexts.

First, taking $\mathcal{C}=$ Top, we prove that the singular and the oriented normalized cubical chain functors are weakly homotopy equivalent (see 4.4.1 for the precise definition) as symmetric monoidal functors, so their extension to topological operads give weak-equivalent $d g$ operads, (see theorem 7.2.2). As a consequence, for a topological operad $P$, the $d g$ operads $S_{*}(P)$ and $C_{*}^{\text {ord }}(P)$ are weakly equivalent, so the formality of $P$ does not depend on the chosen chain functor. This enables us to relate two different versions of Kontsevich formality theorem for the little cube operad, the one proved by Kontsevich in [Ko] for oriented cubical chains and the one by Tamarkin for singular chains (see [T]), and to give a more precise statement of Deligne's conjecture.

The second application refers to a uniqueness result for cohomology theories in the sense of Cartan (see [C]). We obtain a uniqueness result in the category of $d g$ algebras localized with respect to the class of weak equivalences (see theorem 9.1.2 for the precise statement), from which follows the main theorems in Cartan's paper. In particular, for any simplicial set $X$ we deduce that the $\mathbb{Q}$-differential graded algebras of singular cochains $S^{*}(X ; \mathbb{Q})$ and of Sullivan polynomial forms $S u^{*}(X)$ are weakly equivalent, recovering a result of rational homotopy theory, (see [S], [FHT]).

We now give an overview of the contents of the different sections. In section 2 , we recall some basic facts about monoidal functors, and we set some notations used through the paper and review some relevant examples. We begin section 3 by reviewing the Eilenberg-MacLane shuffle 
product formulas and the Alexander-Whitney formulas, which relate the tensor product in the category of complexes with the tensor product in the category of simplicial objects in an additive category $\mathcal{A}$. We use these general formulas to state the existence of several monoidal and comonoidal simple functors. Section 4 is devoted to the statement and proof of theorem 4.3.1. Following Barr, ([B96]), we introduce acyclic classes of complexes, the associated weak equivalences, and the traces of these classes in the category of monoidal functors. Then, given a monoidal cotriple $\mathbf{G}$ in $\mathcal{C}$ we define the relative notions of $\mathbf{G}$-presentable monoidal functors from $\mathcal{C}$ to a category of complexes $\mathbf{C}_{*}(\mathcal{A})$ and of $\mathrm{G}$-weak equivalence for a monoidal natural transformations between these functors, and prove the main theorem.

In section 5, we introduce a suitable monoidal triple $\mathbf{G}$ in the category Top, we prove that singular and normalizad cubical cochains functors are monoidal G-presentable and G-acyclic, and, as a first application of our general result, we establish a comparison theorem between this two monoidal functors. In section 6 , following the same arguments used in the proof of theorem 4.3.1 and corollary 4.3.3, we obtain theorem 6.1.1, which is an acyclic model theorem for symmetric monoidal functors, from which we prove a comparison result for the singular and oriented normalized cubical chains as symmetric monoidal functors, using a triple of Kleisly. As an application we obtain a similar result for topological operads and topological modular operads in section 7 . In particular, we obtain the formality of $C_{*}^{\text {ord }}(\overline{\mathcal{M}}, \mathbb{Q})$, the $d g$ modular operad of cubical chains of the modular operad of Deligne-Knudsen-Mumford moduli spaces of algebraic curves with marked points. Section 8 is devoted to obtain an acyclic models theorem for contravariant monoidal functors, and we apply this result to obtain a comparison result between the singular and cubical cochains functors on Top. Finally, in section 9 we apply the previous result to cohomology theories in the sense of Cartan, including the comparison of Sullivan polynomial forms and singular cochains.

We should point out that in this paper we do not work out the contravariant symmetric case. It will be dealt with elsewhere as an example of a more systematic study of acyclic models in the context of descent categories (see [GN]).

\section{The MONOIDAL BACKGROUND}

In this section we recall the basic principles of monoidal categories and functors, (see [EK] or [ML for details), and introduce notations that are to be used further in the paper. These remarks allow us to define in 3.2 the simple functors which are associated with simplicial and cosimplicial functors with values in a category of complexes.

\subsection{Monoidal categories.}

2.1.1. A monoidal category consists of a category $\mathcal{C}$, a functor $\otimes: \mathcal{C} \times \mathcal{C} \longrightarrow \mathcal{C}$, which we shall call the product functor, and an object $\mathbf{1}$ of $\mathcal{C}$, in addition to natural isomorphisms of associativity for $\otimes$ and unit for $\mathbf{1}$, which are subject to coherence constraints. 
2.1.2. A monoidal category $(\mathcal{C}, \otimes, \mathbf{1})$ is symmetric if, for any objects $X, Y$ of $\mathcal{C}$, it comes equipped with a natural commutative isomorphism $\tau_{X, Y}: X \otimes Y \longrightarrow Y \otimes X$ that satisfies the commutativity constraints.

2.1.3. By an additive monoidal category, also called an additive tensor category, we understand an additive category $\mathcal{A}$ which is monoidal in such a way that the product functor $\otimes: \mathcal{A} \times \mathcal{A} \longrightarrow$ $\mathcal{A}$ is biadditive.

Examples 2.1.1. In these examples we set out the monoidal structure of several categories that will appear later on in this paper.

1. Let $\mathcal{A}$ be an additive category. We denote by $\mathbf{C}_{*}(\mathcal{A})$ the category of uniformly bounded below chain complexes on $\mathcal{A}$, that is, there is an $n \in \mathbb{Z}$ such that the objects of $\mathrm{C}_{*}(\mathcal{A})$ are differential graded objects $\left(C_{*}, d\right)$, with differential $d$ of degree -1 , with $C_{i}=0$ for $i<n$.

If $\mathcal{A}$ is an additive monoidal category, then the category $\mathrm{C}_{*}(\mathcal{A})$ is an additive monoidal category with the product given by $\left(C_{*} \otimes D_{*}\right)_{n}=\oplus_{p+q=n} C_{p} \otimes D_{q}$, the differential on $C_{p} \otimes D_{q}$ being $d=d_{C} \otimes$ id $+(-1)^{p}$ id $\otimes d_{D}$, and the unit given by the complex consisting of the unit of $\mathcal{A}$ concentrated in degree zero.

If $\mathcal{A}$ is a symmetric monoidal category, then $\mathrm{C}_{*}(\mathcal{A})$ is also symmetric, with the natural commutativity isomorphism

$$
\tau_{C_{*}, D_{*}}: C_{*} \otimes D_{*} \longrightarrow D_{*} \otimes C_{*}
$$

that includes the signs, i.e., it is defined by

$$
\tau_{C_{*}, D_{*}}=(-1)^{p q} \tau_{C_{p}, D_{q}}, \quad \text { on } \quad C_{p} \otimes D_{q} .
$$

If $\mathcal{A}$ is the category of $R$-modules for some $\operatorname{ring} R$, we will denote $\mathbf{C}_{*}(\mathcal{A})$ by $\mathbf{C}_{*}(R)$.

2. Let $\mathcal{A}$ be an additive monoidal category. By a double complex of $\mathcal{A}$ we understand a bigraded object of $\mathcal{A}, C_{* *}$, with two differentials, $d^{\prime}, d^{\prime \prime}$, which commute; with the obvious morphisms, they form a category. Thus, the category of uniformly bounded below double chain complexes $\mathbf{C}_{* *}(\mathcal{A})$ is isomorphic to $\mathbf{C}_{*}\left(\mathbf{C}_{*}(\mathcal{A})\right)$.

3. Let $\mathcal{X}$ be a category and $\mathcal{D}$ be a monoidal category. In the functor category $\operatorname{Cat}(\mathcal{X}, \mathcal{D})$ (also denoted by $\operatorname{Fun}(\mathcal{X}, \mathcal{C})$, see [B96]) we define the product $\otimes$ componentwise by

$$
(F \otimes G)(X)=F(X) \otimes G(X),
$$

and the unit 1 as the constant functor defined by the unit of $\mathcal{D}$. With this structure $\operatorname{Cat}(\mathcal{X}, \mathcal{D})$ becomes a monoidal category, which is symmetric if $\mathcal{D}$ is symmetric.

In particular, if $\Delta$ denotes the simplicial category, the category of simplicial objects of $\mathcal{D}$, which we denote by $\Delta^{o} \mathcal{D}$, is a monoidal category. Its objects are denoted by $X$.

Analogously, the category of cosimplicial objects, denoted by $\Delta \mathcal{D}$, is a monoidal category, which is symmetric if $\mathcal{D}$ is symmetric. Its objects will be denoted by $X^{\bullet}$.

4. Observe that if $\mathcal{C}$ is a monoidal category, then the dual category $\mathcal{C}^{o}$ is also monoidal. In particular, if $\mathcal{A}$ is an additive monoidal category, the category of uniformly bounded below cochain complexes $\mathbf{C}^{*}(\mathcal{A})$, which is isomorphic to the dual category of $\mathbf{C}_{*}\left(\mathcal{A}^{o}\right)$, is a monoidal 
category, which is symmetric if $\mathcal{A}$ is symmetric. Likewise the category of uniformly bounded below double chain complexes $\mathbf{C}^{* *}(\mathcal{A})$ is a monoidal category.

\subsection{Monoidal functors.}

2.2.1. If $(\mathcal{C}, \otimes, \mathbf{1}),\left(\mathcal{D}, \otimes, \mathbf{1}^{\prime}\right)$ are monoidal categories, a monoidal functor (sometimes called a lax monoidal functor) is a triple

$$
(F, \kappa, \eta):(\mathcal{C}, \otimes, \mathbf{1}) \longrightarrow\left(\mathcal{D}, \otimes, \mathbf{1}^{\prime}\right),
$$

where $F: \mathcal{C} \longrightarrow \mathcal{D}$ is a covariant functor, together with a natural morphism of $\mathcal{D}$,

$$
\kappa_{X, Y}: F X \otimes F Y \longrightarrow F(X \otimes Y),
$$

for all objects $X, Y$ of $\mathcal{C}$, and a morphism of $\mathcal{D}, \eta: \mathbf{1}^{\prime} \longrightarrow F \mathbf{1}$, compatible with the constraints of associativity and unit. We will refer to $\kappa$ as the Künneth morphism of $F$.

A monoidal functor $F$ is said to be a strong monoidal functor if the Kunneth morphisms are isomorphisms.

2.2.2. If $\mathcal{C}$ and $\mathcal{D}$ are symmetric monoidal categories, a monoidal functor $F: \mathcal{C} \longrightarrow \mathcal{D}$ is said to be symmetric if $\kappa$ is compatible with the commutativity isomorphisms $\tau$.

Examples 2.2.1. Let $\mathcal{A}$ be an additive (symmetric) monoidal category.

1. Given a double complex $C_{* *}$ of $\mathcal{A}$ with finite anti-diagonals, the associated total complex is the complex $\operatorname{Tot}\left(C_{* *}\right)$ which in degree $n$ is given by $\operatorname{Tot}_{n}\left(C_{* *}\right)=\oplus_{p+q=n} C_{p q}$ with differential defined by $d=d^{\prime}+(-1)^{p} d^{\prime \prime}$ on $C_{p q}$. For bounded below double complexes the total complex defines a functor Tot : $\mathbf{C}_{* *}(\mathcal{A}) \longrightarrow \mathbf{C}_{*}(\mathcal{A})$. It is a strong (symmetric) monoidal functor.

Similarly, the total complex functor defines a strong (symmetric) monoidal functor for cochain complexes Tot : $\mathbf{C}^{* *}(\mathcal{A}) \longrightarrow \mathbf{C}^{*}(\mathcal{A})$.

2. The homology functor $H_{*}: \mathbf{C}_{*}(\mathcal{A}) \longrightarrow \mathbf{C}_{*}(\mathcal{A})$ is a (symmetric) monoidal functor, taking as $\kappa$ the usual Künneth morphism

$$
\kappa_{X, Y}: H_{*}(X) \otimes H_{*}(Y) \longrightarrow H_{*}(X \otimes Y) .
$$

2.3. Monoidal functors are stable under composition, so the category of monoidal categories and monoidal functors, Mon, is a subcategory of Cat.

2.3.1. Let $F, G: \mathcal{C} \longrightarrow \mathcal{D}$ be two monoidal functors. A natural transformation $\phi: F \Rightarrow G$ is said to be monoidal if it is compatible with $\kappa$ and $\eta$.

Monoidal functors between two monoidal categories $\mathcal{C}, \mathcal{D}$, together with monoidal natural transformations, define a subcategory of the functor category $\operatorname{Cat}(\mathcal{C}, \mathcal{D})$ which will be denoted by $\operatorname{Mon}(\mathcal{C}, \mathcal{D})$.

Moreover, monoidal functors and monoidal natural transformations are compatible, so they equip the class of monoidal categories, Mon, with a 2-category structure. 
2.3.2. Similarly, one can extend the notions above to the symmetric setting. Hence, the symmetric monoidal functors between two symmetric monoidal categories $\mathcal{C}, \mathcal{D}$, together with symmetric monoidal natural transformations define a subcategory of the functor category $\operatorname{Cat}(\mathcal{C}, \mathcal{D})$ which is denoted by $\operatorname{SyMon}(\mathcal{C}, \mathcal{D})$.

2.4. Comonoidal functors. After the definition of monoidal functor we obtain, by duality, the notion of comonoidal functor between monoidal categories (called op-monoidal functor in $[\mathrm{KS}]$ ).

2.4.1. By definition, a comonoidal functor between $\mathcal{C}$ and $\mathcal{D}$ is a covariant monoidal functor between the dual categories $\mathcal{C}^{o}, \mathcal{D}^{o}$.

That is to say, a comonoidal functor $F: \mathcal{C} \longrightarrow \mathcal{D}$ is a covariant functor together with natural morphisms

$$
\kappa^{o}: F(X \otimes Y) \longrightarrow F(X) \otimes F(Y)
$$

for objects $X, Y$ of $\mathcal{C}$, and a morphism $\eta^{o}: F \mathbf{1} \longrightarrow \mathbf{1}^{\prime}$ of $\mathcal{D}$, satisfying constraints of associativity and unit. If $\mathcal{C}, \mathcal{D}$, are symmetric monoidal categories, a comonoidal functor $F$ is said to be symmetric if $\kappa^{o}$ is compatible with the commutativity constraint.

2.4.2. The definition of comonoidal natural transformations is clear. We denote by $\operatorname{CoMon}(\mathcal{C}, \mathcal{D})$ the category of comonoidal functors and comonoidal natural transformations. The category $\operatorname{CoMon}(\mathcal{C}, \mathcal{D})$ is the dual category of $\operatorname{Mon}\left(\mathcal{C}^{o}, \mathcal{D}^{o}\right)$, so we will focus our attention on monoidal functors.

2.4.3. We have defined the notions of monoidal and comonoidal functors for covariant functors between two monoidal categories $\mathcal{C}, \mathcal{D}$. It is straightforward to define the corresponding notions for contravariant functors from $\mathcal{C}$ to $\mathcal{D}$. Nevertheless, we should point out that a contravariant monoidal functor $\mathcal{C} \longrightarrow \mathcal{D}$ (resp. a contravariant comonoidal functor) is equivalent to a comonoidal functor (resp. monoidal functor) defined in the dual category, $\mathcal{C}^{o} \longrightarrow \mathcal{D}$, so nothing new is gained.

\section{Shuffle And Alexander-Whitney maps}

3.1. Simplicial chain complexes. Let $\mathcal{A}$ be an additive monoidal category. We use $\sim$ : $\Delta^{o} \mathcal{A} \longrightarrow \mathbf{C}_{*}(\mathcal{A})$ to denote the functor which takes a simplicial object $C$. of $\mathcal{A}$ to the chain complex $\widetilde{C_{\bullet}}$ given by $\left(\widetilde{C_{\bullet}}\right)_{n}=C_{n}$ with differential $d=\partial_{0}-\partial_{1}+\cdots+(-1)^{n} \partial_{n}$.

3.1.1. Shuffle map. The functor $\sim$ with the shuffle map is monoidal (see [EM2]): recall that if $C_{\bullet}, D_{\bullet}$ are objects of $\Delta^{o} \mathcal{A}$, the shuffle product $s h: C_{*} \otimes D_{*} \longrightarrow \widehat{C_{\bullet} \otimes D}$. is defined in degree $n=p+q, C_{p} \otimes D_{q} \longrightarrow C_{p+q} \otimes D_{p+q}$, by the formula

$$
s h_{p q}=\sum_{(\mu, \nu)} \varepsilon(\mu, \nu)\left(s_{\nu_{q}} \cdots s_{\nu_{1}}\right) \otimes\left(s_{\mu_{p}} \cdots s_{\mu_{1}}\right),
$$


where the sum is taken over all $(p, q)$-shuffles $(\mu, \nu)$ and $\varepsilon(\mu, \nu)$ is the signature of the associated permutation.

3.1.2. Alexander-Whitney map. The functor $\sim$ with the Alexander-Whitney map is also comonoidal, (loc. cit.). Recall that if $C_{\bullet}, D_{\bullet}$ are simplicial objects of $\mathcal{A}$, the Alexander-Whitney morphism $A W: \widetilde{C_{\bullet} \otimes D_{\bullet}} \longrightarrow C_{*} \otimes D_{*}$ is given by morphisms $A W: C_{n} \otimes D_{n} \longrightarrow \oplus_{i=0}^{n} C_{i} \otimes D_{n-i}$ which are defined by

$$
A W=\sum_{i=0}^{n} \widetilde{\partial}^{n-i} \otimes \partial_{0}^{i},
$$

where $\widetilde{\partial}^{p-i}$ is the last face operator $\partial_{i+1} \ldots \partial_{p}$.

3.1.3. Cosimplicial cochain complexes. Let $\mathcal{A}$ be an additive monoidal category. The category of cosimplicial objects of $\mathcal{A}, \Delta \mathcal{A}$, is a monoidal category. Let $\sim: \Delta \mathcal{A} \longrightarrow \mathrm{C}^{*}(\mathcal{A})$ represent the functor which takes a cosimplicial object $K^{\bullet}$ to the cochain complex $K^{*}$ with differential $d=\partial^{0}+\cdots+(-1)^{n} \partial^{n}$. By dualizing 3.1.1 and 3.1.2, one obtains comonoidal and monoidal structures, respectively, for this functor $\sim$. We use the monoidal structure issuing from the Alexander-Whitney formulas to study contravariant functors in $\S 8$.

3.2. The simple functors. When $\mathcal{A}$ is an additive monoidal category we can use the shuffle and Alexander-Whitney structures above to put monoidal and comonoidal structures in the classic simple complex associated to a simplicial complex of $\mathcal{A}$.

3.2.1. $\quad \mathrm{As}_{*} \mathbf{C}_{*}(\mathcal{A})$ is an additive monoidal category, the functor $\sim$ defines a functor

$$
\Delta^{o} \mathbf{C}_{*}(\mathcal{A}) \longrightarrow \mathbf{C}_{* *}(\mathcal{A}),
$$

which, with the shuffle product, is monoidal. The composition of this functor with the total functor (which, as pointed out in example 2.2.1, is monoidal) is a monoidal functor

$$
s_{E M}: \Delta^{o} \mathbf{C}_{*}(\mathcal{A}) \longrightarrow \mathbf{C}_{*}(\mathcal{A}),
$$

which will be called the Eilenberg-MacLane simple functor (or the simple functor, for short).

3.2.2. If $\mathcal{C}, \mathcal{D}$ are monoidal categories, there is an equivalence of categories $\Delta^{o} \operatorname{Mon}(\mathcal{C}, \mathcal{D}) \cong$ $\operatorname{Mon}\left(\mathcal{C}, \Delta^{o} \mathcal{D}\right)$, so if $\mathcal{A}$ is an additive monoidal category, the composition of this isomorphism with the Eilenberg-MacLane simple functor is a functor, also denoted by $s_{E M}$,

$$
s_{E M}: \Delta^{o} \operatorname{Mon}\left(\mathcal{C}, \mathbf{C}_{*}(\mathcal{A})\right) \longrightarrow \operatorname{Mon}\left(\mathcal{C}, \mathbf{C}_{*}(\mathcal{A})\right),
$$

which associates a monoidal functor to a simplicial monoidal functor between $\mathcal{C}$ and $\mathrm{C}_{*}(\mathcal{A})$. We will also refer to this composition as the Eilenberg-MacLane simple functor. 
3.2.3. If in 3.2.1 we use the comonoidal structure on $\sim$ that comes from the Alexander-Whitney map, we obtain a comonoidal functor

$$
s_{A W}: \Delta^{o} \mathbf{C}_{*}(\mathcal{A}) \longrightarrow \mathbf{C}_{*}(\mathcal{A}),
$$

which we call the Alexander-Whitney simple functor. Thus, in a way completely analogous to 3.2 .2 , we obtain a simple functor for comonoidal functors

$$
s_{A W}: \Delta^{o} \operatorname{CoMon}\left(\mathcal{C}, \mathbf{C}_{*}(\mathcal{A})\right) \longrightarrow \operatorname{CoMon}\left(\mathcal{C}, \mathbf{C}_{*}(\mathcal{A})\right) .
$$

3.2.4. By duality, there are similarly defined simple functors for the categories of monoidal and comonoidal functors with values in the cochain category $\mathbf{C}^{*}(\mathcal{A})$. In $\S 8$ we will use, in the contravariant setting, the Alexander-Whitney simple functor

$$
s_{A W}: \Delta \operatorname{Mon}\left(\mathcal{C}^{o}, \mathbf{C}^{*}(\mathcal{A})\right) \longrightarrow \operatorname{Mon}\left(\mathcal{C}^{o}, \mathbf{C}^{*}(\mathcal{A})\right) .
$$

Although $s_{A W}$ denotes the two functors introduced above, it will be clear from the context which we are referring to.

3.3. Symmetric simple functor. Let $\mathcal{A}$ be an additive symmetric monoidal category. As the shuffle product is symmetric, ([EM2]), the Eilenberg-MacLane simple functor $s_{E M}: \Delta^{o} \mathbf{C}_{*}(\mathcal{A}) \longrightarrow$ $\mathrm{C}_{*}(\mathcal{A})$ is a symmetric monoidal functor. Thus, if $\mathcal{C}$ is a symmetric monoidal category, we can follow the reasoning in 3.2 .2 to deduce the existence of an Eilenberg-MacLane symmetric simple functor

$$
s_{E M}: \Delta^{o} \operatorname{SyMon}\left(\mathcal{C}, \mathbf{C}_{*}(\mathcal{A})\right) \longrightarrow \operatorname{SyMon}\left(\mathcal{C}, \mathbf{C}_{*}(\mathcal{A})\right)
$$

\section{AN ACYCLIC MODELS THEOREM FOR MONOIDAL FUNCTORS}

In this section we prove the main technical tool, the acyclic models theorem for monoidal functors. Our presentation is a variation of the scheme devised by Barr in [B96] for these kinds of results. According to Barr, three main ingredients are required to state an acyclic models theorem: the total complex associated to a double complex, the acyclic classes of complexes that define the associated classes of weak equivalences, and the cotriples constructed from the models. The total complex functor and its monoidal counterparts were the object of the previous section, we begin now by introducing acyclic classes.

4.1. Acyclic classes and weak equivalences. Let $\mathcal{A}$ be an abelian category. If $C_{*}$ is a chain complex on $\mathcal{A}$, we denote by $C_{*}[-1]$ the chain complex given by $\left(C_{*}[-1]\right)_{n}=C_{n-1}$ with differential defined by $-d_{n-1}$.

4.1.1. Recall the following definition from [B96], (see also [B02]),

Definition 4.1.1. A class $\Gamma$ of complexes in $\mathbf{C}_{*}(\mathcal{A})$ is called an acyclic class if the following conditions are satisfied:

(AC1) The complex 0 is in $\Gamma$.

(AC2) Stability: $C_{*}$ is in $\Gamma$ if and only if $C_{*}[-1]$ is in $\Gamma$.

(AC3) Let $C_{*}, D_{*}$ be two chain homotopic complexes. Then $C_{*}$ is in $\Gamma$ if and only if $D_{*}$ is in $\Gamma$.

(AC4) Every complex in $\Gamma$ is acyclic. 
(AC5) If $C_{* *}$ is a double complex in $\mathbf{C}_{* *}(\mathcal{A})$ all of whose rows are in $\Gamma$, then the total complex Tot $\mathrm{C}_{* *}$ is in $\Gamma$.

By (AC1) and (AC3) every contractible complex is in $\Gamma$ and by (AC4) all complexes in $\Gamma$ are acyclic, thus $\Gamma$ is a class between the class of all contractible complexes and the class of all acyclic complexes. These two extreme cases are examples of acyclic classes, (see [B96], §4).

4.1.2. Given an acyclic class $\Gamma$ in $\mathbf{C}_{*}(\mathcal{A})$, we denote by $\Sigma$ the class of morphisms between chain complexes of $\mathcal{A}$ whose mapping cone is in $\Gamma$. Morphisms in $\Sigma$ are referred to as weak equivalences (with respect to $\Gamma$ ).

By (AC1) and (AC3) every homotopy equivalence is in $\Sigma$ and by (AC4) the mapping cone of a morphism $f$ of $\Sigma$ is acyclic, thus it follows from the exact sequence of the mapping cone that any such $f$ is a quasi-isomorphism.

Remark 4.1.2. Let $\mathbf{K}_{*}(\mathcal{A})$ be the category of chain complexes up to homotopy, that is, its objects are complexes of $\mathcal{A}$ and its morphisms are homotopy classes of morphisms of complexes. It is a triangulated category. An acyclic class $\Gamma$ determines a triangulated subcategory of $\mathbf{K}_{*}(\mathcal{A})$ and, as a consequence, the class of morphisms $\Sigma$ associated with $\Gamma$ inherits some properties from the general setup of triangulated categories, (i.e. it admits a calculus of fractions). For the sake of simplicity we will follow Barr's treatment and refer to [B95] for the properties of $\Sigma$ that will be used.

4.1.3. We use $\mathbf{C}_{*}(\mathcal{A})\left[\Sigma^{-1}\right]$ to denote the localized category of $\mathbf{C}_{*}(\mathcal{A})$ with respect to $\Sigma$, which exists in a suitable universe, (see [GZ]), and is uniquely determined up to isomorphism.

The class of morphisms $\Sigma$ is stable under composition and satisfies the 2 out of 3 property (see the proof of proposition 3.3 in [B96]), that is, for every pair of morphisms $f, g$ of $\mathbf{C}_{*}(\mathcal{A})$ so that $g f$ exists, if two of $f, g$ and $g f$ are in $\Sigma$, then so is the third. Moreover, $\Sigma$ has a homotopy calculus of fractions (see loc. cit.). In many cases, such as when $\Sigma$ is the class of quasi-isomorphisms or the class of homotopy equivalences, $\Sigma$ is a saturated class of morphisms, in other words, it is precisely the class of morphisms of $\mathbf{C}_{*}(\mathcal{A})$ which become isomorphisms in $\mathbf{C}_{*}(\mathcal{A})\left[\Sigma^{-1}\right]$.

Two objects $C_{*}, D_{*}$ of $\mathbf{C}_{*}(\mathcal{A})$ are said to be weakly equivalent (with respect to $\Gamma$ ) if there exists a sequence of morphisms of $\mathbf{C}_{*}(\mathcal{A})$,

$$
C_{*} \longleftarrow C_{*}^{1} \longrightarrow \ldots \longleftarrow C_{*}^{m} \longrightarrow D_{*},
$$

which are weak equivalences. Hence, weakly equivalent objects are isomorphic in $\mathbf{C}_{*}(\mathcal{A})\left[\Sigma^{-1}\right]$.

4.1.4. Let $\mathcal{C}$ be a category and $\mathcal{A}$ an abelian category. The functor category $\operatorname{Cat}(\mathcal{C}, \mathcal{A})$ is an abelian category. We fix a class $\Sigma$ of weak equivalences in $\operatorname{Cat}\left(\mathcal{C}, \mathbf{C}_{*}(\mathcal{A})\right)$ associated to an acyclic class $\Gamma$ in $\mathbf{C}_{*}(\operatorname{Cat}(\mathcal{C}, \mathcal{A}))=\operatorname{Cat}\left(\mathcal{C}, \mathbf{C}_{*}(\mathcal{A})\right)$.

Let us now assume that $\mathcal{C}$ is a monoidal category and $\mathcal{A}$ is an abelian monoidal category. As $\operatorname{Mon}\left(\mathcal{C}, \mathbf{C}_{*}(\mathcal{A})\right)$ is a subcategory of $\operatorname{Cat}\left(\mathcal{C}, \mathbf{C}_{*}(\mathcal{A})\right)$, $\Sigma$ determines a class of morphisms in $\operatorname{Mon}\left(\mathcal{C}, \mathbf{C}_{*}(\mathcal{A})\right)$, which will be represented by the same symbol. Thus, a morphism of $\operatorname{Mon}\left(\mathcal{C}, \mathbf{C}_{*}(\mathcal{A})\right)$ is a weak equivalence if it is in $\Sigma$ as a morphism of $\operatorname{Cat}\left(\mathcal{C}, \mathbf{C}_{*}(\mathcal{A})\right)$. 
However, note that for two monoidal functors from $\mathcal{C}$ to $\mathrm{C}_{*}(\mathcal{A})$, the weak equivalence relation in $\operatorname{Mon}\left(\mathcal{C}, \mathbf{C}_{*}(\mathcal{A})\right)$ is not the same as it is in $\operatorname{Cat}\left(\mathcal{C}, \mathbf{C}_{*}(\mathcal{A})\right)$, since in the first case the intermediate functors in the chain 4.1 .3 have to be monoidal.

We use $\operatorname{Mon}\left(\mathcal{C}, \mathbf{C}_{*}(\mathcal{A})\right)\left[\Sigma^{-1}\right]$ to denote the category obtained by inverting the weak equivalences in $\Sigma$, so the natural functor $\operatorname{Mon}\left(\mathcal{C}, \mathbf{C}_{*}(\mathcal{A})\right) \longrightarrow \operatorname{Mon}\left(\mathcal{C}, \mathbf{C}_{*}(\mathcal{A})\right)\left[\Sigma^{-1}\right]$ transforms weak equivalences to isomorphisms.

4.1.5. We note that the class of weak equivalences in $\operatorname{Mon}\left(\mathcal{C}, \mathbf{C}_{*}(\mathcal{A})\right)$ contains the homotopy equivalences and is compatible with the functor $s_{E M}$, so it defines a functor

$$
s_{E M}:\left(\Delta^{o} \operatorname{Mon}\left(\mathcal{C}, \mathbf{C}_{*}(\mathcal{A})\right)\right)\left[\widetilde{\Sigma}^{-1}\right] \longrightarrow \operatorname{Mon}\left(\mathcal{C}, \mathbf{C}_{*}(\mathcal{A})\right)\left[\Sigma^{-1}\right],
$$

where $\widetilde{\Sigma}$ is the class of morphisms in $\Delta^{o} \operatorname{Mon}\left(\mathcal{C}, \mathbf{C}_{*}(\mathcal{A})\right)$ which in each simplicial degree are in $\Sigma$, as follows from (AC5).

This compatibility is one of the basic properties of acyclic classes that is needed to prove the acyclic models theorem below. Instead of Barr's acyclic classes we could work in other settings where there is such a compatibility (see also 4.5.). 
4.2. G-presentable objects and G-weak equivalences. Let $\mathcal{C}$ be a monoidal category, $\mathcal{A}$ an abelian monoidal category, $\Sigma$ a class of weak equivalences in $\operatorname{Cat}\left(\mathcal{C}, \mathbf{C}_{*}(\mathcal{A})\right)$ which contains the homotopy equivalences and is compatible with $s_{E M}$.

4.2.1. The standard construction. Recall that if $\mathcal{X}$ is a category, a cotriple (also called a comonad, see [ML]) $\mathbf{G}=(G, \varepsilon, \delta)$ in $\mathcal{X}$ is given by a functor $G: \mathcal{X} \longrightarrow \mathcal{X}$ and natural transformations $\varepsilon: G \Rightarrow$ id and $\delta: G \Rightarrow G^{2}$, which satisfies

$$
\delta G \cdot \delta=G \delta \cdot \delta: G \Rightarrow G^{3}, \quad \varepsilon G \cdot \delta=\mathbf{1}_{G}=G \varepsilon \cdot \delta: G \Rightarrow G,
$$

where the dot denotes the composition of natural transformations, (see [ML]). Given a cotriple $\mathrm{G}$ in $\mathcal{X}$, every object $X$ of $\mathcal{X}$ has a functorial augmented simplicial object associated with it, $B .(X)$, which will be called the standard construction of $\mathbf{G}$ applied to $X$, ([ML], chapter VII). In degree $n, n \geq 0$, the simplicial object $B \cdot(X)$ is given by $G^{n+1}(X)$ with face and degeneracy transformations given by

$$
\begin{array}{ll}
\partial_{i}=G^{i} \varepsilon G^{n-i}: G^{n+1}(X) \longrightarrow G^{n}(X), & 0 \leq i \leq n, \\
s_{i}=G^{i} \delta G^{n-i}: G^{n+1}(X) \longrightarrow G^{n+2}(X), & 0 \leq i \leq n .
\end{array}
$$

In this way we obtain a simplicial object with an augmentation defined by $\varepsilon: B_{0}(X)=$ $G(X) \longrightarrow X$.

By the functoriality of the standard construction we obtain an augmented simplicial functor $B$. in $\operatorname{Cat}(\mathcal{X}, \mathcal{X})$.

Proposition 4.2.1. (1) The augmented simplicial functor $G(\varepsilon): G \circ B_{\bullet} \Rightarrow G$ is contractible.

(2) The augmented simplicial functor $G(\varepsilon): B \bullet \circ \Rightarrow G$ is contractible.

Proof. (1) The face and degeneracy morphisms of $G \circ B_{\bullet}$ are given by

$$
\begin{aligned}
& G\left(\partial_{i}^{n}\right)=G^{i+1} \varepsilon G^{n-i}=\partial_{i+1}^{n+1}, \quad 0 \leq i \leq n, \\
& G\left(s_{i}^{n}\right)=G^{i+1} \delta G^{n-i}=s_{i+1}^{n+1}, \quad 0 \leq i \leq n,
\end{aligned}
$$

where the latter are the faces and degeneracies of $B_{\bullet}$. Now, the extra degeneracy from $B_{\bullet}$, $s=s_{0}^{n+1}$, gives a contraction for $G \circ B_{\bullet}$.

For (2) use the last extra degeneracy $s_{n+1}^{n+1}$ in $B_{n} \circ G$.

4.2.2. Monoidal structure in the standard construction. Let $\mathbf{G}$ be a cotriple in $\operatorname{Mon}\left(\mathcal{C}, \mathbf{C}_{*}(\mathcal{A})\right)$. We say that $\mathbf{G}$ is compatible with $\Sigma$ if $G(\Sigma) \subseteq \Sigma$. We will associate to $\mathbf{G}$ a monoidal functor $B_{*}: \operatorname{Mon}\left(\mathcal{C}, \mathbf{C}_{*}(\mathcal{A})\right) \longrightarrow \operatorname{Mon}\left(\mathcal{C}, \mathbf{C}_{*}(\mathcal{A})\right)$.

The standard construction defines a functor

$$
B_{\bullet}: \operatorname{Mon}\left(\mathcal{C}, \mathbf{C}_{*}(\mathcal{A})\right) \longrightarrow \Delta^{o} \operatorname{Mon}\left(\mathcal{C}, \mathbf{C}_{*}(\mathcal{A})\right) \text {. }
$$

By composing $B \bullet$ with the Eilenberg-MacLane simple functor defined in $3.2 .2, s_{E M}$, we obtain a functor

$$
B_{*}=s_{E M} B_{\bullet}: \operatorname{Mon}\left(\mathcal{C}, \mathbf{C}_{*}(\mathcal{A})\right) \longrightarrow \operatorname{Mon}\left(\mathcal{C}, \mathbf{C}_{*}(\mathcal{A})\right) .
$$

The natural transformation $\varepsilon$ gives a natural transformation $\varepsilon: B_{*} \Rightarrow$ id. 
4.2.3. G-presentable objects. Barr introduces in [B96] the $\varepsilon$-presentable objects. We will refer to these objects as $\mathbf{G}$-presentable objects. Recall its definition:

Definition 4.2.2. We say that $K_{*}$ of $\operatorname{Mon}\left(\mathcal{C}, \mathbf{C}_{*}(\mathcal{A})\right.$ ) is $\mathbf{G}$-presentable (with respect to $\Gamma$ ) if the augmentation morphism $\varepsilon: B_{*}\left(K_{*}\right) \longrightarrow K_{*}$ is a weak equivalence.

As remarked in 4.1.5, $\Sigma$ contains the homotopy equivalences, so if $K_{*}$ is an object of $\operatorname{Mon}\left(\mathcal{C}, \mathbf{C}_{*}(\mathcal{A})\right)$ such that $\varepsilon_{K_{n}}$ splits for all $n$, then $\varepsilon_{K_{n}} \in \Sigma$, and since $\Sigma$ is compatible with $s_{E M}$, it follows that $\varepsilon_{K} \in \Sigma$. Hence we obtain the following result,

Proposition 4.2.3. Let $K_{*}$ be an object of $\operatorname{Mon}\left(\mathcal{C}, \mathbf{C}_{*}(\mathcal{A})\right)$ such that $\varepsilon_{K_{n}}$ splits for all $n \in \mathbb{Z}$, that is to say, for each $n$ there is a natural transformation $\theta_{n}: K_{n} \longrightarrow K_{n} G$ such that $\varepsilon_{K_{n}} \theta_{n}=$ id. Then, $K_{*}$ is $\mathbf{G}$-presentable.

Example 4.2.4. If $K_{*}$ is an object of $\operatorname{Mon}\left(\mathcal{C}, \mathbf{C}_{*}(\mathcal{A})\right)$, then $G\left(K_{*}\right)$ is $\mathbf{G}$-presentable, since for each $n$ we can split $G\left(K_{n}\right)$ by $\delta$.

4.2.4. G-Weak equivalences. Barr introduces in [B96] the $\mathbf{G}$-acyclic objects. More generally we can speak of objects that are $\mathbf{G}$-equivalent in the following sense,

Definition 4.2.5. Let $f: K_{*} \longrightarrow L_{*}$ be a morphism of $\operatorname{Mon}\left(\mathcal{C}, \mathbf{C}_{*}(\mathcal{A})\right)$. We say that $f$ is a BG-weak equivalence, (respectively, a G-weak equivalence), with respect to $\Gamma$, if $B_{*}(f) \in \Sigma$, (respectively, if $G(f) \in \Sigma$ ).

Proposition 4.2.6. If $G(\Sigma) \subseteq \Sigma$, then a morphism $f: K_{*} \longrightarrow L_{*}$ of $\operatorname{Mon}\left(\mathcal{C}, \mathbf{C}_{*}(\mathcal{A})\right)$ is a $\mathbf{G}$-weak equivalence if, and only if, it is a BG-weak equivalence.

Proof. If $f$ is a G-weak equivalence then, for all $n \geq 0, G^{n+1}(f)$ is a weak equivalence, by hypothesis. Therefore, by (AC5), $f$ is a $B \mathrm{G}$-weak equivalence.

Reciprocally, if we assume that $f$ is a $B$ G-weak equivalence, then $G(B(f)): G\left(B_{*}\left(K_{*}\right)\right) \longrightarrow$ $G\left(B_{*}\left(L_{*}\right)\right)$ is a weak equivalence, by hypothesis. Consider the commutative diagram

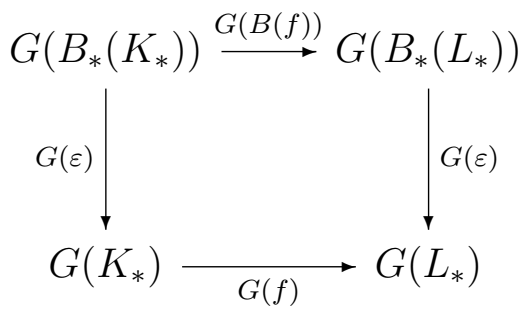

By proposition 4.2.1 (2), the two vertical morphisms are isomorphisms, so the result follows from the 3 out of 2 property of $\Sigma$.

Now we recover Barr's definition:

Definition 4.2.7. An object $K_{*}$ of $\operatorname{Mon}\left(\mathcal{C}, \mathbf{C}_{*}(\mathcal{A})\right.$ ), in non-negative degrees, (that is, with $K_{p}=0$ if $p<0$ ), is said to be $\mathbf{G}$-acyclic (with respect to $\Gamma$ ) if the augmentation $K_{*} \longrightarrow H_{0}\left(K_{*}\right.$ ) is a $\mathbf{G}$-weak equivalence. 
4.3. The acyclic models theorem for monoidal functors. The following result and its corollaries are a variation of the classical acyclic models theorem in the context of monoidal functors (see [EM1] and also [BB], [B96]).

Let $\mathcal{C}$ be a monoidal category, $\mathcal{A}$ an abelian monoidal category, and $\Sigma$ a class of weak equivalences in $\operatorname{Mon}\left(\mathcal{C}, \mathbf{C}_{*}(\mathcal{A})\right)$ which contains the homotopy equivalences and is compatible with $s_{E M}$. Let $\mathbf{G}$ be a cotriple in $\operatorname{Mon}\left(\mathcal{C}, \mathbf{C}_{*}(\mathcal{A})\right)$.

Theorem 4.3.1. Let $K_{*}$ be a $\mathbf{G}$-presentable object of $\operatorname{Mon}\left(\mathcal{C}, \mathbf{C}_{*}(\mathcal{A})\right)$ and $s: L_{*} \longrightarrow M_{*} a$ G-weak equivalence. Suppose that $\mathbf{G}$ is compatible with $\Sigma$, (that is, $G(\Sigma) \subseteq \Sigma$ ). Then, for any $\alpha: K_{*} \longrightarrow M_{*}$ there exists a unique morphism $\widetilde{\alpha}: K_{*} \longrightarrow L_{*}$ in $\operatorname{Mon}\left(\mathcal{C}, \mathbf{C}_{*}(\mathcal{A})\right)\left[\Sigma^{-1}\right]$ such that $\alpha=s \widetilde{\alpha}$ in this localized category.

Proof. By the naturality of $\varepsilon$, we have a commutative diagram

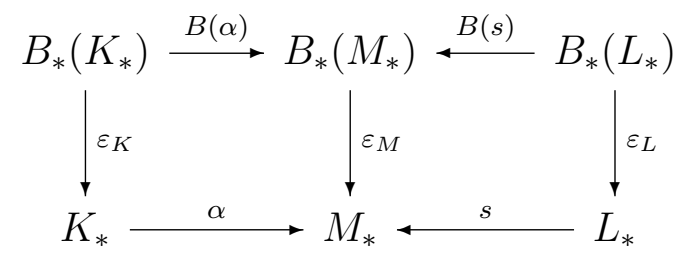

As $K_{*}$ is $\mathbf{G}$-presentable, $\varepsilon_{K}$ is a weak equivalence. Moreover, by proposition 4.2.6, $B(s)$ is also a weak equivalence. So they are isomorphisms in the localized category $\operatorname{Mon}\left(\mathcal{C}, \mathbf{C}_{*}(\mathcal{A})\right)\left[\Sigma^{-1}\right]$.

In this category we define the morphism

$$
\widetilde{\alpha}=\varepsilon_{L} B(s)^{-1} B(\alpha)\left(\varepsilon_{K}\right)^{-1} .
$$

We have $s \widetilde{\alpha}=\alpha$, since by the commutativity of the diagram above we have $\alpha=\varepsilon_{M} B(\alpha)\left(\varepsilon_{K}\right)^{-1}$ and $\varepsilon_{M}=s \varepsilon_{L} B(s)^{-1}$, so it follows that

$$
\alpha=\varepsilon_{M} B(\alpha)\left(\varepsilon_{K}\right)^{-1}=s \varepsilon_{L} B(s)^{-1} B(\alpha)\left(\varepsilon_{K}\right)^{-1}=s \widetilde{\alpha} .
$$

With respect to uniqueness, assume that $\gamma: K_{*} \longrightarrow L_{*}$ is another lifting of $\alpha$ in $\operatorname{Mon}\left(\mathcal{C}, \mathbf{C}_{*}(\mathcal{A})\right)\left[\Sigma^{-1}\right]$, so that $\alpha=s \gamma$. Since the standard construction is functorial and compatible with weak equivalences because $G(\Sigma) \subseteq \Sigma$, we have $B(s) B(\widetilde{\alpha})=B(\alpha)=B(s) B(\gamma)$, but $B(s) \in \Sigma$ so $B(\gamma)=B(\widetilde{\alpha})$. Moreover, as $\varepsilon$ is a natural transformation, we have $\varepsilon_{L} B(\gamma)=\gamma \varepsilon_{K}$ and $\varepsilon_{L} B(\widetilde{\alpha})=\widetilde{\alpha} \varepsilon_{K}$, and since $\varepsilon_{K} \in \Sigma$ we deduce that $\gamma=\widetilde{\alpha}$. This ends the proof of the theorem.

Corollary 4.3.2. Let $K_{*}, L_{*}$ be objects of $\operatorname{Mon}\left(\mathcal{C}, \mathbf{C}_{*}(\mathcal{A})\right)$ in non-negative degrees. Suppose that $K_{*}$ is $\mathbf{G}$-presentable and $L_{*}$ is $\mathbf{G}$-acyclic. Then, any monoidal natural transformation $H_{0}\left(K_{*}\right) \longrightarrow H_{0}\left(L_{*}\right)$ has a unique extension to a morphism $K_{*} \longrightarrow L_{*}$ in $\operatorname{Mon}\left(\mathcal{C}, \mathbf{C}_{*}(\mathcal{A})\right)\left[\Sigma^{-1}\right]$.

Proof. It follows from the previous theorem applied to the diagram

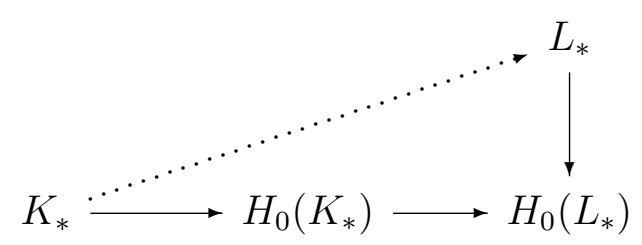


Corollary 4.3.3. Let $K_{*}, L_{*}$ be objects of $\operatorname{Mon}\left(\mathcal{C}, \mathbf{C}_{*}(\mathcal{A})\right)$ in non-negative degrees. Suppose that $K_{*}, L_{*}$ are $\mathbf{G}$-acyclic and $\mathbf{G}$-presentable. Then any monoidal natural isomorphism $H_{0}\left(K_{*}\right) \longrightarrow$ $H_{0}\left(L_{*}\right)$ lifts to a unique isomorphism $f_{*}: K_{*} \longrightarrow L_{*}$ in $\mathbf{M o n}\left(\mathcal{C}, \mathbf{C}_{*}(\mathcal{A})\right)\left[\Sigma^{-1}\right]$.

4.4. Weak homotopy type. In the following sections we will apply the general results above to some chain-valued functors defined in the category of topological spaces or in the category of simplicial sets. In all cases, the acyclic classes in $\mathbf{C}_{*}(\operatorname{Cat}(\mathcal{C}, \mathcal{A}))$ will come from acyclic classes in $\mathbf{C}_{*}(\mathcal{A})$, and the cotriples in $\mathbf{C}_{*}(\operatorname{Cat}(\mathcal{C}, \mathcal{A}))$ will come from monoidal cotriples in $\mathcal{C}$. Let us describe the main features of this situation.

4.4.1. Let $\Gamma$ be an acyclic class in $\mathbf{C}_{*}(\mathcal{A})$ and $\Sigma$ the associated class of weak equivalences. We extend $\Sigma$ to a class of weak equivalences $\widetilde{\Sigma}$ in $\mathbf{C}_{*}(\operatorname{Cat}(\mathcal{X}, \mathcal{A}))$ componentwise: a morphism $f: K_{*} \longrightarrow L_{*}$ is in $\widetilde{\Sigma}$ if, and only if, for all objects $X$ of $\mathcal{C}, f_{X}: K_{*}(X) \longrightarrow L_{*}(X)$ is in $\Sigma$.

If we take the contractible complexes in $\mathbf{C}_{*}(\mathcal{A})$ as the acyclic class $\Gamma$, we will say that $\widetilde{\Gamma}$ is the acyclic class of weakly contractible functors in $\mathbf{C}_{*}(\operatorname{Cat}(\mathcal{X}, \mathcal{A}))$. The morphisms in $\widetilde{\Sigma}$ will be called weak homotopy equivalences. If $\Gamma$ is the class of acyclic complexes, we will say that $\Sigma$ is the class of weak quasi-isomorphisms.

4.4.2. Monoidal cotriples. Given a monoidal category $\mathcal{C}$, a monoidal cotriple in $\mathcal{C}$ is a cotriple $\mathbf{G}=(G, \varepsilon, \delta)$, such that $G: \mathcal{C} \longrightarrow \mathcal{C}$ is a monoidal functor and $\varepsilon: G \Rightarrow \mathrm{id}_{\mathcal{C}}$ and $\delta: G \Rightarrow G^{2}$ are monoidal natural transformations.

If $\mathbf{G}=(G, \varepsilon, \delta)$ is a monoidal cotriple in $\mathcal{C}$, define a functor

$$
\widetilde{G}: \operatorname{Mon}\left(\mathcal{C}, \mathbf{C}_{*}(\mathcal{A})\right) \longrightarrow \operatorname{Mon}\left(\mathcal{C}, \mathbf{C}_{*}(\mathcal{A})\right),
$$

by composition $\widetilde{G}\left(F_{*}\right)=F_{*} G$, and let $\widetilde{\varepsilon}: \widetilde{G} \Rightarrow$ id and $\widetilde{\delta}: \widetilde{G} \Rightarrow \widetilde{G}^{2}$ be the natural transformations induced by $\varepsilon, \delta$. It follows easily from the definitions that $\widetilde{\mathbf{G}}=(\widetilde{G}, \widetilde{\varepsilon}, \widetilde{\delta})$ is a cotriple in $\operatorname{Mon}\left(\mathcal{C}, \mathbf{C}_{*}(\mathcal{A})\right)$.

Proposition 4.4.1. Let $\Gamma$ be an acyclic class in $\mathbf{C}_{*}(\mathcal{A})$ and $\mathbf{G}$ a monoidal cotriple in $\mathcal{C}$. Consider the class of morphisms $\widetilde{\Sigma}$ and the cotriple $\widetilde{\mathbf{G}}$ induced in $\mathbf{M o n}\left(\mathcal{C}, \mathbf{C}_{*}(\mathcal{A})\right)$. Then, $\widetilde{\mathbf{G}}$ is compatible with $\widetilde{\Sigma}$, that is, $\widetilde{G}(\widetilde{\Sigma}) \subseteq \widetilde{\Sigma}$.

Proof. If $f: K_{*} \longrightarrow L_{*}$ is in $\widetilde{\Sigma}$, then $f_{X}: K_{*}(X) \longrightarrow L_{*}(X)$, for all objects $X$ of $\mathcal{C}$. In particular, $f_{G(X)} \in \Sigma$, thus $\widetilde{G}(f) \in \widetilde{\Sigma}$.

4.5. A detailed analysis of the proof of the acyclic models theorem shows that, in fact, the main ingredients of our proof are a monoidal functor $s_{E M}: \Delta^{o} \mathbf{C}_{*}(\mathcal{A}) \longrightarrow \mathbf{C}_{*}(\mathcal{A})$ and a saturated class of morphisms $\Sigma$ of $\mathbf{C}_{*}(\mathcal{A})$, such that

(1) $\Sigma$ contains the homotopy equivalences.

(2) If $f_{* *}: C_{* *} \longrightarrow D_{* *}$ is a morphism of double complexes such that $f_{n *}$ is in $\Sigma$, then $s_{E M}\left(f_{* *}\right)$ is also in $\Sigma$. 
We will analyze this more general situation elsewhere.

\section{Application: COMPARison of Singular And CUbical CHains}

In this section we compare the singular and cubical chains of a topological space in the monoidal setting by applying the results above, extending the well-known classic comparison theorem, [EM1].

5.1. Let Top denote the category of topological spaces, which is a monoidal category under cartesian product, and let $\mathbf{C}_{*}(\mathbb{Z})$ be the category of chain complexes of abelian groups. We fix the class $\Sigma$ of weak homotopy equivalences in the functor category $\operatorname{Cat}\left(\operatorname{Top}, \mathbf{C}_{*}(\mathbb{Z})\right)$, 4.4.1.

It is well known, EM1, that the functor of singular chains

$$
S_{*}: \text { Top } \longrightarrow \mathbf{C}_{*}(\mathbb{Z}),
$$

together with the shuffle product as the Künneth morphism is a monoidal functor.

We can also consider the functor of cubical chains,

$$
C_{*}: \operatorname{Top} \longrightarrow \mathrm{C}_{*}(\mathbb{Z}) .
$$

For this we take Massey's notations ([Mas]): for a topological space $X$, let $Q_{p}(X)$ be the free abelian group generated by the continuous maps $I^{p} \longrightarrow X$, where $I$ is the unit interval of the real line. Its elements are the cubical chains of $X$. Now, $C_{n}(X)$ is defined as the quotient of $Q_{n}(X)$ modulo the degenerated chains. Together with the cross product

$$
\times: C_{*}(X) \otimes C_{*}(Y) \longrightarrow C_{*}(X \times Y),
$$

which for singular cubes $c: I^{p} \longrightarrow X$ and $d: I^{q} \longrightarrow Y$ is defined as the cartesian product

$$
c \times d: I^{p+q}=I^{p} \times I^{q} \longrightarrow X \times Y,
$$

$C_{*}$ is a monoidal functor.

5.2. A monoidal cotriple in Top. In order to compare $S_{*}$ and $C_{*}$ as monoidal functors following corollary 4.3.3, we introduce a monoidal cotriple in Top. It is a model induced cotriple, (see [B02], §4.2).

In the classical version of the acyclic models theorem the models are the standard simplexes $\Delta^{m}, m \geq 0$. To obtain a monoidal version we take products of these spaces.

Therefore, for any sequence $\underline{n}=\left(n_{1}, \ldots, n_{r}\right), n_{i} \geq 0$, take $\Delta^{\underline{n}}=\Delta^{n_{1}} \times \cdots \times \Delta^{n_{r}}$. We define $\mathbf{G}=(G, \varepsilon, \delta)$ as the model induced cotriple with models $\Delta^{n}$. That is, the functor $G:$ Top $\longrightarrow$ Top takes a topological space $X$ to

$$
G(X)=\bigsqcup_{\alpha: \Delta^{\underline{n}} \rightarrow X}\left(\Delta^{\underline{n}}, \alpha\right)
$$

where $\left(\Delta^{\underline{n}}, \alpha\right)$ is a copy of $\Delta^{\underline{n}}$ indexed by the continuous map $\alpha: \Delta^{\underline{n}} \rightarrow X$ and where the disjoint union is over all sequences $\underline{n}$ and maps $\alpha$. G takes a continuous map $f: X \longrightarrow Y$, to 
the map

$$
G(f): \bigsqcup_{\alpha: \Delta^{\underline{n}} \rightarrow X}\left(\Delta^{\underline{n}}, \alpha\right) \longrightarrow \bigsqcup_{\beta: \Delta^{\underline{n}} \rightarrow Y}\left(\Delta^{\underline{n}}, \beta\right),
$$

which is the identity from $\left(\Delta^{\underline{n}}, \alpha\right)$ to $\left(\Delta^{\underline{n}}, f \circ \alpha\right)$.

For a topological space $X$ define the map

$$
\varepsilon_{X}: G(X)=\bigsqcup_{\alpha: \Delta^{\underline{n}} \rightarrow X}\left(\Delta^{\underline{n}}, \alpha\right) \longrightarrow X,
$$

which over $\left(\Delta^{\underline{n}}, \alpha\right)$ is $\alpha: \Delta^{\underline{n}} \longrightarrow X$, so we have a natural transformation $\varepsilon: G \Rightarrow$ id. Finally we define a natural transformation $\delta: G \Rightarrow G^{2}$ : the iteration of $G$ gives the functor

$$
G^{2}(X)=\bigsqcup_{\Delta \underline{m} \stackrel{\beta}{\longrightarrow} \Delta_{\underline{n}} \stackrel{\alpha}{\longrightarrow} X}\left(\left(\Delta^{\underline{m}}, \alpha\right), \beta\right)
$$

so we can define $\delta: G(X) \longrightarrow G^{2}(X)$ as the identity from $\left(\Delta^{\underline{n}}, \alpha\right)$ to $\left(\left(\Delta^{\underline{n}}, \alpha\right)\right.$, id).

Moreover, this cotriple has a monoidal structure. To define the Künneth morphisms of $G$ consider topological spaces $X, Y$ and take the map

$\kappa_{X, Y}: G(X) \times G(Y)=\left(\bigsqcup_{\alpha: \Delta \underline{n} \rightarrow X}\left(\Delta^{\underline{n}}, \alpha\right)\right) \times\left(\bigsqcup_{\beta: \Delta \underline{\underline{m}} \rightarrow Y}\left(\Delta^{\underline{m}}, \beta\right)\right) \rightarrow G(X \times Y)=\bigsqcup_{\gamma: \Delta^{\underline{r}} \rightarrow X \times Y}\left(\Delta^{\underline{r}}, \gamma\right)$,

given by

$$
\left(\Delta^{\underline{n}}, \alpha\right) \times\left(\Delta^{\underline{m}}, \beta\right) \stackrel{\text { id }}{\longrightarrow}\left(\Delta^{\underline{n}} \times \Delta^{\underline{m}}, \alpha \times \beta\right) .
$$

It is straightforward to prove that $\mathbf{G}$ is a monoidal cotriple in Top.

5.3. As in previous sections, we denote also by $\mathbf{G}=(G, \varepsilon, \delta)$ the cotriple induced in $\mathbf{M o n}\left(\mathbf{T o p}, \mathbf{C}_{*}(\mathbb{Z})\right)$ by the monoidal cotriple in Top defined above.

Theorem 5.3.1. The singular and cubical chain monoidal functors $S_{*}, C_{*}:$ Top $\longrightarrow \mathbf{C}_{*}(\mathbb{Z})$ are weakly homotopy equivalent monoidal functors, that is, they are weakly equivalent in $\mathbf{M o n}\left(\mathbf{T o p}, \mathbf{C}_{*}(\mathbb{Z})\right)$.

Proof. Both $H_{0} S_{*}$ and $H_{0} C_{*}$ are the functors that associate to a topological space $X$ the free group generated by the points of $X$, so the result will follow from corollary 4.3 .3 after we prove that $S_{*}, C_{*}$ are $\mathbf{G}$-presentable and $\mathbf{G}$-acyclic as objects of $\mathbf{M o n}\left(\mathbf{T o p}, \mathbf{C}_{*}(\mathbb{Z})\right)$.

With respect to G-acyclicity for $S_{*}$ we have to prove that $G\left(S_{*}\right) \longrightarrow G\left(H_{0}\right)$ is a weak equivalence. That is to say, that the morphisms

$$
\bigoplus_{\alpha: \Delta \underline{n} \longrightarrow X} S_{*}\left(\Delta^{\underline{n}}\right) \longrightarrow \bigoplus_{\alpha: \Delta \underline{n} \longrightarrow X} \mathbb{Z}
$$

where in each summand the morphism $S_{*}\left(\Delta^{\underline{n}}\right) \longrightarrow \mathbb{Z}$ is the natural augmentation, are homotopy equivalences, for all $X$. As $\Delta^{n}$ are contractible spaces, the result is clear. Similarly, we can prove the G-acyclicity of $C_{*}$.

To prove G-presentability it is sufficient, by proposition 4.2.3, to prove that $\varepsilon_{C_{n}}$ and $\varepsilon_{S_{n}}$ split, for all $n \geq 0$. We can define a natural transformation

$$
\theta_{S}: S_{n} \longrightarrow G\left(S_{n}\right),
$$


by sending a singular simplex $\sigma: \Delta^{n} \longrightarrow X$ on the topological space $X$ to the simplex of $G(X)$ given by id : $\Delta^{n} \longrightarrow\left(\Delta^{n}, \sigma\right)$. It follows from the definition that $\varepsilon \circ \theta_{S}=\mathrm{id}$, so this morphism splits the standard resolution $B_{*}\left(S_{*}\right)$.

For the cubical chains, observe that $I^{n}$ is homeomorphic to $\Delta^{1} \times \stackrel{n}{n} . \times \Delta^{1}$, so that a $n$-cube on a space $X$ is given by a map $\Delta^{1} \times \stackrel{n)}{.} \times \Delta^{1} \longrightarrow X$. We can now follow the definition of $\theta_{S}$ to define a natural transformation for cubical chains

$$
\theta_{Q}: Q_{n} \longrightarrow G\left(Q_{n}\right),
$$

by sending a cubical simplex $c: \Delta^{1} \times \stackrel{n)}{.} \times \Delta^{1} \longrightarrow X$ to the cubical simplex of $G(X)$ given by id : $\Delta^{1} \times \stackrel{n}{.} . \times \Delta^{1} \longrightarrow\left(\Delta^{1} \times \stackrel{n}{.} . \times \Delta^{1} \longrightarrow X, c\right)$. This natural transformation $\theta_{Q}$ splits the augmentation $\varepsilon_{Q_{n}}$. But the natural projection $\pi_{n}: Q_{n} \longrightarrow C_{n}$ admits a section $\nu_{n}, \pi_{n} \nu_{n}=\mathrm{id}$, (see [Mas], lemma 5.5), so we can define $\theta_{C}=\theta_{Q} \nu_{n}$, which splits $\varepsilon_{C_{n}}$, as is easily verified.

\section{Symmetric MONOIDAL FUNCTORS}

In this section we indicate how to extend the results of the previous sections to symmetric monoidal functors. We will focus our attention on the symmetric version of corollary 4.3.3. which we will apply to obtain a comparison result in the symmetric setting for the singular and ordered cubical functors acting on topological spaces.

6.1. Acyclic models for symmetric monoidal functors. Let $\mathcal{C}$ be a symmetric monoidal category, $\mathcal{A}$ a symmetric abelian monoidal category, $\Gamma$ an acyclic class in $\mathbf{C}_{*}(\mathbf{C a t}(\mathcal{C}, \mathcal{A}))$ and $\Sigma$ the class of weak equivalences. $\Sigma$ determines a class of morphisms in $\operatorname{SyMon}\left(\mathcal{C}, \mathbf{C}_{*}(\mathcal{A})\right)$, which will be represented by the same symbol. Let $\mathbf{G}$ be a cotriple in $\operatorname{SyMon}\left(\mathcal{C}, \mathbf{C}_{*}(\mathcal{A})\right)$ compatible with $\Sigma$, i.e., $G(\Sigma) \subseteq \Sigma$.

Following the general procedure in 4.2 .1 , given $\mathbf{G}$ in $\operatorname{SyMon}\left(\mathcal{C}, \mathbf{C}_{*}(\mathcal{A})\right)$, we can associate a functor

$$
B_{\bullet}: \operatorname{SyMon}\left(\mathcal{C}, \mathbf{C}_{*}(\mathcal{A})\right) \longrightarrow \Delta^{o} \operatorname{SyMon}\left(\mathcal{C}, \mathbf{C}_{*}(\mathcal{A})\right),
$$

in such a way that the simplicial object $B_{\bullet}\left(K_{*}\right)$ is augmented to $K_{*}$ by $\varepsilon$. By composing this functor with the symmetric simple functor, 3.3 , we obtain a functor

$$
B_{*}: \operatorname{SyMon}\left(\mathcal{C}, \mathbf{C}_{*}(\mathcal{A})\right) \longrightarrow \operatorname{SyMon}\left(\mathcal{C}, \mathbf{C}_{*}(\mathcal{A})\right)
$$

The natural transformation $\varepsilon$ gives a natural transformation $B_{*} \Rightarrow$ id.

We can now reproduce the definitions and results from section $\S 4$ in the symmetric setting. In particular, we have the following result, which is analogous to corollary 4.3.3.

Theorem 6.1.1. Let $K_{*}, L_{*}$ be objects of $\operatorname{SyMon}\left(\mathcal{C}, \mathbf{C}_{*}(\mathcal{A})\right)$ in non-negative degrees. Suppose that $K_{*}, L_{*}$ are $\mathbf{G}$-acyclic and $\mathbf{G}$-presentable. Then any symmetric monoidal natural isomorphism $H_{0}\left(K_{*}\right) \longrightarrow H_{0}\left(L_{*}\right)$ lifts to a unique isomorphism $f_{*}: K_{*} \longrightarrow L_{*}$ in $\operatorname{SyMon}\left(\mathcal{C}, \mathbf{C}_{*}(\mathcal{A})\right)\left[\Sigma^{-1}\right]$.

A symmetric monoidal cotriple $\mathbf{G}=(G, \varepsilon, \delta)$ on a symmetric monoidal category $\mathcal{C}$ is a monoidal cotriple such that $G$ is a symmetric monoidal functor and $\varepsilon, \delta$ are monoidal transformations. As in 4.4.2, if $\mathcal{D}$ is another symmetric monoidal category, a symmetric monoidal cotriple on $\mathcal{C}$ 
induces a cotriple on $\operatorname{SyMon}(\mathcal{C}, \mathcal{D})$, that will also be denoted by $\mathbf{G}$, which satisfies $G(\Sigma) \subseteq \Sigma$ (see proposition 4.4.1).

6.2. The Kleisli cotriple. The cotriple in Top defined in 5.2 is not symmetric. Therefore in order to apply theorem 6.1.1 with $\mathcal{C}=$ Top we must first define a suitable symmetric monoidal cotriple on Top. The following cotriple, introduced by Kleisli in [K2], will turn out to be symmetric and monoidal: for a topological space $X$ and an element $x \in X$, let $P(X, x)$ denote the space of pointed continuous paths $\alpha:(I, 0) \longrightarrow(X, x)$, which is topologized by the compact-open topology. Then, define $G$ on $X$ by

$$
G(X)=\bigsqcup_{x \in X} P(X, x)
$$

so $G(X)$ is the set of paths in $X$, but with a topology that is not the path space topology. Nevertheless, note that if $Z$ is a connected space, a map from $Z$ to $G(X)$ is equivalent to a map $\beta: Z \times I \longrightarrow X$ such that $\beta(z, 0)=x$, for some $x \in X$ and all $z \in Z$. The action of $G$ on a continuous map $f: X \longrightarrow Y$ is given by composition, that is, if $\alpha \in P(X, x)$, then $G(f)(\alpha)=f \circ \alpha$.

Define a natural transformation $\varepsilon: G \Rightarrow$ id by evaluating paths at 1 . Finally, note that the iteration $G^{2}$ over a space $X$ is given by

$$
G^{2}(X)=\bigsqcup_{x \in X} \bigsqcup_{\alpha \in P(X, x)} P(P(X, x), \alpha) .
$$

An element of $P(P(X, x), \alpha)$ is determined by a map $\widetilde{\alpha}: I^{2} \longrightarrow X$ with $\widetilde{\alpha}(t, 0)=\alpha(t)$, for all $t \in I$, and $\widetilde{\alpha}(0, s)=x$, for all $s \in I$. We define the natural transformation $\delta: G \Rightarrow G^{2}$ by maps $P(X, x) \longrightarrow P(P(X, x), x)$ that send $\alpha$ to the map $\widetilde{\alpha}(t, s)=\alpha(t s)$.

The cotriple $\mathbf{G}=(G, \varepsilon, \delta)$ will be called the Kleisli cotriple. It is a symmetric monoidal: for topological spaces $X, Y$ define the Künneth morphism

$$
\kappa_{X, Y}: G(X) \times G(Y)=\bigsqcup_{x \in X} P(X, x) \times \bigsqcup_{y \in Y} P(Y, y) \longrightarrow G(X \times Y)=\bigsqcup_{(x, y) \in X \times Y} P(X \times Y,(x, y)),
$$

by sending the paths $\alpha \in P(X, x)$ and $\beta \in P(Y, y)$ to the path $\alpha \times \beta \in P(X \times Y,(x, y))$. It is evident that these morphisms are compatible with the symmetric structure of Top.

6.3. Ordered cubical chains. The cubical chain functor is not symmetric. However, the ordered cubical chains define a symmetric monoidal functor that may be compared directly with $S_{*}$.

6.3.1. The ordered cubical chains of a topological space $X$ are defined as follows (see [Ko]). If $c: I^{n} \longrightarrow X$ is a singular $n$-cube and $\pi \in \Sigma_{n}$ define the chain $\pi c$ as

$$
(\pi c)\left(t_{1}, \ldots, t_{n}\right)=\varepsilon(\pi) c\left(t_{\pi(0)}, \ldots, t_{\pi(n)}\right),
$$

and extend this action to $C_{n}(X)$ linearly. Let $D_{n}(X)$ be the subgroup of $C_{n}(X)$ generated by chains of the form $c-\pi c$. Then $D_{*}(X)$ is a subcomplex of $C_{*}(X)$, so we can define the ordered 
cubical chains of $X$ as the quotient complex

$$
C_{*}^{\text {ord }}(X)=C_{*}(X) / D_{*}(X) .
$$

There is a natural transformation $C_{*} \Rightarrow C_{*}^{\text {ord }}$.

6.3.2. The monoidal structure of the cubical chain functor $C_{*}$ with the usual cross product is carried over the quotient by $D_{*}$. In fact, if $X, Y$ are topological spaces, the cross product maps $C_{*}(X) \otimes D_{*}(Y)$ to $D_{*}(X \times Y)$ since if $c \in C_{p}(X), d \in C_{q}(Y)$ are singular cubes and $\pi \in \Sigma_{p}$, then

$$
(c-\pi c) \times d=(c \times d)-\tilde{\pi}(c \times d),
$$

where $\widetilde{\pi}$ is the element of $\Sigma_{p+q}$ that acts as $\pi$ on the first $p$ elements and fixes the rest. Analogously, $\times$ maps $D_{*}(X) \otimes C_{*}(Y)$ to $D_{*}(X \times Y)$.

Proposition 6.3.1. The functor $C_{*}^{\text {ord }}$ : Top $\longrightarrow \mathbf{C}_{*}(\mathbb{Z})$ is a symmetric monoidal functor and the natural transformation $C_{*} \Rightarrow C_{*}^{o r d}$ is a monoidal natural transformation.

Proof. The proof follows immediately from the definitions. Note that the cross product in $C_{*}^{\text {ord }}$ is symmetric, since if $X, Y$ are spaces and $c \otimes d \in C_{p}^{\text {ord }}(X) \otimes C_{q}^{\text {ord }}(X)$, then

$$
\begin{aligned}
\tau(c \otimes d)\left(t_{1}, \ldots, t_{p+q}\right) & =(-1)^{p q}(d \otimes c)\left(t_{1}, \ldots, t_{p+q}\right) \\
& =(d \otimes c)\left(t_{q}, \ldots, t_{p+q}, t_{1}, \ldots, t_{p}\right) \\
& =\tau_{X, Y}(c \times d)\left(t_{1}, \ldots, t_{p+q}\right),
\end{aligned}
$$

where in the second equality we used the invariance of the oriented cubical chains by the action of the symmetric group.

6.3.3. We fix in $\operatorname{SyMon}\left(\mathbf{T o p}, \mathbf{C}_{*}(\mathbb{Z})\right)$ the class $\Sigma$ of weak homotopy equivalences, that is, the symmetric monoidal functors $f$ such that $f_{X}$ is a homotopy equivalence for each space $X$. Let G be the cotriple induced by the Kleisli cotriple, which satisfies $G(\Sigma) \subseteq \Sigma$, see 4.4.1.

Theorem 6.3.2. The singular and ordered cubical chain functors $S_{*}, C_{*}^{\text {ord }}:$ Top $\longrightarrow \mathbf{C}_{*}(\mathbb{Z})$ are weakly homotopy equivalent symmetric monoidal functors.

Proof. For a topological space $X$, both groups $H_{0}\left(S_{*}(X)\right)$ and $H_{0}\left(C_{*}^{\text {ord }}(X)\right)$ are isomorphic to the free group generated by the points of $X$, so the result will follow from theorem 6.1.1 after we prove that $S_{*}$ and $C_{*}^{\text {ord }}$ are $\mathbf{G}$-presentable and $\mathbf{G}$-acyclic with respect to the Kleisli cotriple. This has been proved by Barr (see [B02]) for the singular chains functor. Let us prove it for the ordered cubical chains.

Firstly we prove the G-acyclicity of $C_{*}^{o r d}$. We will prove that there is a chain contraction $s$ for the complex $C_{*} G \longrightarrow H_{0} G$ such that for any singular $n$-cube $c$ and any $\sigma \in \Sigma_{n}, s(\sigma c)=\sigma s(c)$.

Let $X$ be any topological space and $c: I^{n} \longrightarrow G(X)$ a singular $n$-cube. By the connectedness of the standard cube, there is a point $x \in X$ such that the map $c$ factors through a map $c: I^{n} \longrightarrow P(X, x)$. By adjunction, $c$ is equivalent to a map $\widetilde{c}: I^{n+1} \longrightarrow X$, which satisfies $\widetilde{c}\left(t_{1}, \ldots, t_{n}, 0\right)=x$, for $\left(t_{1}, \ldots, t_{n}\right) \in I^{n}$. Taking into account the product decomposition $I^{n+1}=$ $I^{n} \times I$, we will write the value of $\widetilde{c}$ at the point $\left(t_{1}, \ldots, t_{n}\right) \in I^{n}$ and $u \in I$ by $\widetilde{c}\left(t_{1}, \ldots, t_{n} ; u\right)$. 
Note that $H_{0}(G(X))$ is the free group generated by the elements of $X$, since the spaces $P(X, x)$ are contractible, and define $s: H_{0}(G(X)) \longrightarrow C_{0}(G(X))$ by $s(x)=p_{x}$, where $p_{x}$ denotes the constant path at $x$.

For $n \geq 0$ define $\mu: I^{n+1} \times I \longrightarrow I^{n} \times I$ by

$$
\mu\left(t_{1}, \ldots, t_{n+1} ; u\right)=\left(t_{1}, \ldots, t_{n} ; t_{n+1} u\right)
$$

and define $s: Q_{n}(G(X)) \longrightarrow Q_{n+1}(G(X))$ by

$$
s(\widetilde{c})=(-1)^{n+1} \widetilde{c} \circ \mu .
$$

Next, we recall the definition of the differential $d: Q_{n}(X) \longrightarrow Q_{n-1}(X)$ of the cubical chain complex: for $1 \leq i \leq n, \epsilon \in\{0,1\}$, let $\delta_{i}^{\epsilon}: I^{n-1} \longrightarrow I^{n}$ denote the face defined by $\delta_{i}^{\epsilon}\left(t_{1}, \ldots, t_{n-1}\right)=\left(t_{1}, \ldots, \epsilon, \ldots, t_{n-1}\right)$, where $\epsilon$ is in the $i$-th place; if $c \in Q_{n}(X), d(c)$ is defined by

$$
d(c)=\sum_{i, \epsilon}(-1)^{i+\epsilon} c \circ \delta_{i}^{\epsilon}
$$

It is clear that on $Q_{n+1}$ we have $\delta_{i}^{\varepsilon} \circ \mu=\mu \circ \delta_{i}^{\varepsilon}$, for $1 \leq i \leq n$, and that

$$
\begin{aligned}
\left(\mu \circ \delta_{n+1}^{0}\right)\left(t_{1}, \ldots, t_{n} ; u\right) & =\left(t_{1}, \ldots, t_{n}, 0\right), \\
\left(\mu \circ \delta_{n+1}^{1}\right)\left(t_{1}, \ldots, t_{n} ; u\right) & =\left(t_{1}, \ldots, t_{n} ; u\right),
\end{aligned}
$$

thus, an easy calculation proves that

$$
(d s+s d)(\widetilde{c})=\widetilde{c}-x .
$$

As $s$ sends degenerated chains to degenerated chains, it defines a map $s: C_{n}(G(X)) \longrightarrow$ $C_{n+1}(G(X))$, and as $x$ represents the constant path $p_{x}$, which is degenerated, the equality above reduces to $d s+s d=$ id in $C_{*}(G(X))$.

The contraction $s$ is trivially compatible with the action of the symmetric group, so it defines a contraction for $C_{*}^{\text {ord }}$, and as a consequence $C_{*}^{\text {ord }}$ is $\mathbf{G}$-acyclic.

To prove G-presentability, notice that the natural transformation $\theta_{n}: Q_{n} \longrightarrow Q_{n} G$ given by

$$
\theta_{n}(\sigma)\left(t_{1}, \ldots, t_{n}\right)(s)=c\left(s t_{1}, \ldots, s t_{n}\right),
$$

is a section of $\varepsilon_{Q}$ which is compatible with taking quotients modulo degenerated chains and the action of the symmetric group $\Sigma_{n}$, so it defines a section $\theta_{n}^{\text {ord }}: C_{n}^{\text {ord }} \longrightarrow C_{n}^{\text {ord }} G$, and we can apply proposition 4.2 .3 .

Remark 6.3.3. Note that if $S_{*}^{N}$ : Top $\longrightarrow \mathbf{C}_{*}(\mathbb{Z})$ denotes the functor of normalized singular chains, which is also a symmetric monoidal functor since the degenerated singular chains are invariant by shuffle product, (see [EM2]), then the projection $S_{*} \Rightarrow S_{*}^{N}$ is a monoidal natural transformation of symmetric monoidal functors which is a weak equivalence. This is a classical result and follows directly from the fact that the degenerated singular chains are a direct factor of singular chains.

\section{Applichtion to operads}

7.1. Operads. Let us recall some definitions and notations about operads (see [MSS]). 
7.1.1. Let $\Sigma$ be the symmetric groupoid, that is, the category whose objects are the sets $\{1, \ldots, n\}, n \geq 1$, and the only morphisms are those of the symmetric groups $\Sigma_{n}$.

7.1.2. Let $\mathcal{C}$ be a monoidal category. The category of contravariant functors from $\Sigma$ to $\mathcal{C}$ is called the category of $\boldsymbol{\Sigma}$-modules and is represented by $\boldsymbol{\Sigma} \operatorname{Mod}_{\mathcal{C}}$. We identify its objects with sequences of objects in $\mathcal{C}, E=\left((E(l))_{l>1}\right.$, with a right $\Sigma_{l}$-action on each $E(l)$. If $E$ and $F$ are $\boldsymbol{\Sigma}$-modules, a morphism of $\boldsymbol{\Sigma}$-modules $f: E \longrightarrow F$ is a sequence of $\Sigma_{l^{-}}$equivariant morphisms $f(l): E(l) \longrightarrow F(l), l \geq 1$.

7.1.3. A unital $\boldsymbol{\Sigma}$-operad (an operad for short) in $\mathcal{C}$ is a $\boldsymbol{\Sigma}$-module $P$ together with a family of composition morphisms

$$
\gamma_{l ; m_{1}, \ldots, m_{l}}: P(l) \otimes P\left(m_{1}\right) \otimes \cdots \otimes P\left(m_{l}\right) \longrightarrow P\left(m_{1}+\cdots+m_{l}\right),
$$

and a unit morphism

$$
\eta: \mathbf{1} \longrightarrow P(1)
$$

which satisfies the axioms of equivariance, associativity and unit. A morphism of operads is a morphism of $\boldsymbol{\Sigma}$-modules that is compatible with structure morphisms. We use $\mathbf{O p}_{\mathcal{C}}$ to denote the category of operads in $\mathcal{C}$ and its morphisms.

An operad in Top is called a topological operad. If $R$ is a ring, an operad in $\mathbf{C}_{*}(R)$ is called a $d g$ operad. We are especially interested in the cases $R=\mathbb{Z}, \mathbb{Q}$.

7.2. We can now extend the comparison result between $S_{*}$ and $C_{*}^{\text {ord }}$ to topological operads.

7.2.1. If $F: \mathcal{C} \longrightarrow \mathcal{D}$ is a symmetric monoidal functor between monoidal categories, it is easy to prove that, applied componentwise, $F$ induces a functor between the corresponding categories of operads

$$
\mathrm{Op}_{F}: \mathrm{Op}_{\mathcal{C}} \longrightarrow \mathrm{Op}_{\mathcal{D}}
$$

also denoted by $F$. Therefore, singular and cubical ordered chains induce functors

$$
S_{*}, C_{*}^{\text {ord }}: \mathrm{Op}_{\text {Tор }} \longrightarrow \mathrm{Op}_{\mathbf{C}_{*}(\mathbb{Z})} \text {. }
$$

Moreover, if $\mathcal{D}$ has a notion of weak equivalence, it extends to $\mathbf{O p}_{\mathcal{D}}$ componentwise. For instance, we can consider the weak equivalence relation in the category of chain complexes $\mathbf{C}_{*}(R)$ induced by quasi-isomorphisms. Now it easily follows from the definitions that

Proposition 7.2.1. Let $\mathcal{C}$ be a monoidal category, $R$ a ring and $F, G \in \operatorname{Mon}\left(\mathcal{C}, \mathbf{C}_{*}(R)\right)$. If $F, G$ are weakly equivalent (with respect to quasi-isomorphism in $\mathbf{C}_{*}(R)$ ), then the functors

$$
\mathrm{Op}_{F}, \mathrm{Op}_{G}: \mathrm{Op}_{\mathcal{C}} \longrightarrow \mathrm{Op}_{\mathrm{C}_{*}(R)}
$$

are weakly equivalent.

This proposition together with theorem 6.3 .2 applied to $S_{*}, C_{*}^{\text {ord }}$ gives 
Theorem 7.2.2. The functors

$$
S_{*}, C_{*}^{\text {ord }}: \mathbf{O p}_{\text {Top }} \longrightarrow \mathrm{Op}_{\mathbf{C}_{*}(\mathbb{Z})}
$$

are weakly equivalent (with respect to quasi-isomorphism). In particular, for a topological operad $P$ the $d g$ operads $S_{*}(P), C_{*}^{\text {ord }}(P)$ are weakly equivalent.

If $\mathrm{HoO} \mathbf{p}_{\mathbf{C}_{*}(\mathbb{Z})}$ denotes the localization of $\mathbf{O} \mathbf{p}_{\mathbf{C}_{*}(\mathbb{Z})}$ with respect to quasi-isomorphisms, we obtain Corollary 7.2.3. There is an isomorphism of functors

$$
S_{*} \cong C_{*}^{\text {ord }}: \mathbf{O p}_{\text {Top }} \longrightarrow \mathrm{HoO}_{\mathbf{C}_{*}(\mathbb{Z})} \text {. }
$$

7.2.2. Theorem 7.2 .2 may be applied to compare the categories of $S_{*}(P)$ and $C_{*}^{\text {ord }}(P)$ algebras up to homotopy.

Recall that, given a dg operad $P$, a $P$-algebra is a chain complex $V$ of finite type together with a morphism $P \longrightarrow \mathcal{E}[V]$, where $\mathcal{E}[V]$ denotes the operad of endomorphisms of $V$. Following GNPR, definition 7.3.1, define a $P$-algebra up to homotopy as a finite type complex $V$ together with a morphism $P \longrightarrow \mathcal{E}[V]$ in $\mathrm{HoOp}_{\mathbf{C}_{*}(\mathbb{Z})}$. From theorem 7.2 .2 , it immediately follows that

Corollary 7.2.4. Let $P$ be a topological operad. The categories of $S_{*}(P)$ and $C_{*}^{\text {ord }}(P)$ algebras up to homotopy are equivalent.

This remark applies to Deligne's conjecture, which is commonly expressed as follows: let $A$ be an associative algebra over a ring $R$, and let $C^{*}(A ; A)$ be the associated Hochschild complex.

Deligne's conjecture. For any associative $R$-algebra $A$, the complex $C^{*}(A ; A)$ is naturally an algebra over the singular chains of the little discs operad $\mathcal{D}_{2}$ or a suitable version of it.

There is some ambiguity in this statement with reference to the chain model of $\mathcal{D}_{2}$ to be used and whether obtaining a solution for one model means that a solution is obtained for any other model. From our results it follows that there is only one chain model of a topological operad up to homotopy, therefore, this ambiguity disappears when we work with structures up to homotopy, linking in this way the solutions of Deligne's conjecture given by Kontsevich, who used $C_{*}^{\text {ord }}\left(\mathcal{D}_{2}\right)$, (see [Ko]), and MacClure-Smith, Tamarkin and Voronov, who used singular chains (see, for example, [T]).

7.3. Formality. Recall that a dg operad $P$ is formal if it is quasi-isomorphic to its homology operad $H(P)$, (see [MSS]). From theorem 7.2 .2 we can deduce the following:

Corollary 7.3.1. Let $P$ be a topological operad. Then $S_{*}(P ; \mathbb{Q})$ is a formal operad if, and only if, $C_{*}^{\text {ord }}(P ; \mathbb{Q})$ is a formal operad.

M. Kontsevich proved the formality of the ordered cubical chains of the little $k$-discs operad after tensoring by the real numbers, $\mathbb{R}$, (see $[\mathrm{Ko}$, theorem 2). The independent nature of formality on the base field proved in GNPR implies that $C_{*}^{\text {ord }}\left(\mathcal{D}_{k} ; \mathbb{Q}\right)$ is also formal over the rational field, so by corollary 7.3.1, $S_{*}\left(\mathcal{D}_{k} ; \mathbb{Q}\right)$ is formal. 
7.4. Modular operads. The results above may be extended to chain models of modular operads. We refer to [GK] and [MSS] for the definitions concerning modular operads. Given a symmetric monoidal category we denote by $\mathbf{M O p}_{\mathcal{C}}$ the category of modular operads of $\mathcal{C}$.

As for operads, it follows from the definitions that every symmetric monoidal functor $F: \mathcal{C} \longrightarrow$ $\mathcal{D}$ applied componentwise induces a functor

$$
\mathrm{MOp}_{F}: \mathrm{MOp}_{\mathcal{C}} \longrightarrow \mathrm{MOp}_{\mathcal{D}}
$$

In particular, the singular and cubical chain functors of topological spaces extend to functors defined in the category of topological modular operads. Now, as in theorem 7.2 .2 , one has

Theorem 7.4.1. The functors

$$
S_{*}, C_{*}^{\text {ord }}: \mathrm{MOp}_{\text {Top }} \longrightarrow \mathrm{MOp}_{\mathbf{C}_{*}(\mathbb{Z})}
$$

are weakly equivalent. In particular, for a topological modular operad $P$, the $d g$ modular operads $S_{*}(P), C_{*}^{o r d}(P)$ are weakly equivalent.

From theorem 7.4.1 it immediately follows that

Proposition 7.4.2. Let $P$ be a topological modular operad. Then $S_{*}(P ; \mathbb{Q})$ is a formal modular operad if and only if $C_{*}^{o r d}(P ; \mathbb{Q})$ is a formal modular operad.

We can apply this result to the modular operad $\mathcal{M}$ : the family $\overline{\mathcal{M}}((g, l))=\overline{\mathcal{M}}_{g, l}$ of DeligneKnudsen-Mumford moduli spaces of stable genus $g$ algebraic curves with $l$ marked points, with the maps that identify marked points, is a modular operad in the category of projective smooth Deligne-Mumford stacks, GK]. In [GNPR we proved that $S_{*}(\overline{\mathcal{M}} ; \mathbb{Q})$ is a formal modular operad, so any other chain model is also formal. For instance, for cubical chains we can state that

Corollary 7.4.3. The $d g$ modular operad $C_{*}^{\text {ord }}(\overline{\mathcal{M}} ; \mathbb{Q})$ is a formal modular operad.

\section{Contravariant FunCtors}

If we work in the category of contravariant monoidal functors between monoidal categories, $\operatorname{Mon}\left(\mathcal{C}^{o}, \mathcal{D}\right)$, a cotriple on $\mathcal{C}$ induces a triple on $\operatorname{Mon}\left(\mathcal{C}^{o}, \mathcal{D}\right)$, so that the standard construction produces cosimplicial objects instead of simplicial objects. As remarked in 2.4 .3 this can be avoided by identifying such functors with covariant comonoidal functors between $\mathcal{C}$ and $\mathcal{D}^{o}$. However, for functors with values in $\mathbf{C}^{*}(\mathbb{Z})$, which are the functors that appear in the study of the cohomology of topological spaces, the dual category $\mathbf{C}^{*}(\mathbb{Z})^{o}$ is not the same as $\mathbf{C}^{*}(\mathbb{Z})$, so the acyclic models theorem 4.3.3 has to be appropriately dualized to cover this case.

In this section we present the minor modifications of the constructions and results of $\S 4$ that are necessary to cover this situation, and we apply them to compare the singular and cubical cochain functors. 
8.1. The standard construction. Let $\mathbf{T}=(T, \eta, \mu)$ be a triple (or monad, see [ML]) in a category $\mathcal{X}$. There is a functor associated to $\mathbf{T}$, which we call the standard construction,

$$
B^{\bullet}: \mathcal{X} \longrightarrow \Delta \mathcal{X}
$$

defined by $B^{n}(X)=T^{n+1}(X)$ and with faces and degeneracies defined analogously as in 4.2.1.

8.1.1. Let $\mathcal{C}$ be a monoidal category and $\mathcal{A}$ an additive monoidal category, and $\Sigma$ a class of weak equivalences of $\operatorname{Mon}\left(\mathcal{C}^{o}, \mathbf{C}^{*}(\mathcal{A})\right)$ which contains the homotopy equivalences and is compatible with the Alexander-Whitney functor $s_{A W}$. A monoidal cotriple $\mathbf{G}=(G, \varepsilon, \delta)$ in $\mathcal{C}$ induces a triple $\mathbf{T}$ on $\mathcal{X}=\operatorname{Mon}\left(\mathcal{C}^{o}, \mathbf{C}^{*}(\mathcal{A})\right)$, whose endofunctor is given by $T\left(K^{*}\right)=K^{*} G$, and with $\eta, \mu$ induced by $\varepsilon, \delta$. By the dual of proposition 4.4.1. $\mathbf{T}$ is compatible with $\Sigma$, that is, $T(\Sigma) \subseteq \Sigma$.

We can compose the standard cosimplicial construction with the Alexander-Whitney simple functor defined in 3.2 .4 to obtain a functor

$$
B^{*}: \operatorname{Mon}\left(\mathcal{C}^{o}, \mathbf{C}^{*}(\mathcal{A})\right) \longrightarrow \operatorname{Mon}\left(\mathcal{C}^{o}, \mathbf{C}^{*}(\mathcal{A})\right) .
$$

Moreover, the natural transformation $\varepsilon$ of $\mathbf{G}$ defines a natural transformation $\varepsilon$ : id $\Rightarrow B^{*}$.

8.2. Acyclic models for contravariant monoidal functors. By analogy to section $\S 4$, it is clear how to define the notions of $\mathbf{T}$-presentability and $\mathbf{T}$-equivalence in $\operatorname{Mon}\left(\mathcal{C}^{o}, \mathbf{C}^{*}(\mathcal{A})\right)$, so that we have all the ingredients to transpose the proof of 4.3 .1 and its corollaries to the category of contravariant monoidal functors between $\mathcal{C}$ and $\mathbf{C}^{*}(\mathcal{A})$. In particular, we can state the contravariant version of corollary 4.3 .3 referred to the assumptions made in the previous paragraph:

Theorem 8.2.1. Let $K^{*}, L^{*}$ be objects of $\operatorname{Mon}\left(\mathcal{C}^{o}, \mathbf{C}^{*}(\mathcal{A})\right)$ concentrated in non-negative degrees, and $\mathbf{T}$ a triple in $\mathbf{M o n}\left(\mathcal{C}^{\circ}, \mathbf{C}^{*}(\mathcal{A})\right)$ induced by a monoidal cotriple $\mathbf{G}$ on $\mathcal{C}$. Suppose that $K^{*}, L^{*}$ are $\mathbf{T}$-acyclic and $\mathbf{T}$-presentable. Then any monoidal transformation $H^{0}\left(K^{*}\right) \longrightarrow H^{0}\left(L^{*}\right)$ lifts to a unique morphism $K^{*} \longrightarrow L^{*}$ in $\operatorname{Mon}\left(\mathcal{C}^{o}, \mathbf{C}^{*}(\mathcal{A})\right)\left[\Sigma^{-1}\right]$. In particular, if $H^{0}\left(K^{*}\right)$ and $H^{0}\left(L^{*}\right)$ are isomorphic, then $K^{*}$ and $L^{*}$ are weakly homotopy equivalent.

8.3. Application to singular and cubical cochains. For a topological space $X$, let $S^{*}(X), C^{*}(X)$ denote, respectively, the complexes of singular and of cubical cochains defined on $X$ :

$$
\begin{aligned}
S^{*}(X) & =\operatorname{Hom}\left(S_{*}(X), \mathbb{Z}\right), \\
C^{*}(X) & =\operatorname{Hom}\left(C_{*}(X), \mathbb{Z}\right) .
\end{aligned}
$$

They define contravariant functors

$$
S^{*}, C^{*}: \mathbf{T o p}^{o} \longrightarrow \mathbf{C}^{*}(\mathbb{Z}) .
$$

By dualizing the Alexander-Whitney morphism for $S_{*}, S^{*}$ becomes a contravariant monoidal functor. There is also an explicit Alexander-Whitney associative morphism for cubical chains $C_{*}(X \times Y) \longrightarrow C_{*}(X) \otimes C_{*}(Y)$ (see [Mas], XI§5 and exercise XIII.5.1), and as a consequence $C^{*}$ is also a contravariant monoidal functor.

The monoidal cotriple $\mathbf{G}$ on Top defined in 5.2 induces a triple in $\mathbf{M o n}\left(\mathbf{T o p}^{o}, \mathbf{C}^{*}(\mathbb{Z})\right)$, denoted by $\mathbf{T}$, and it is easily seen (compare 5.3.1) that $S^{*}$ and $C^{*}$ are $\mathbf{T}$-presentable and $\mathbf{T}$-acyclic. Thus we are able to apply theorem 8.2.1 and deduce: 
Theorem 8.3.1. The singular and cubical cochain functors $S^{*}, C^{*}: \mathbf{T o p}^{o} \longrightarrow \mathbf{C}^{*}(\mathbb{Z})$ are weakly homotopy equivalent contravariant monoidal functors, that is, they are weakly homotopy equivalent in $\mathbf{M o n}\left(\mathbf{T o p}^{o}, \mathbf{C}^{*}(\mathbb{Z})\right)$.

\section{Applichtion to COHOMOlogy theories}

In this section we apply the acyclic models theorem 8.2.1 to compare cohomology theories on simplicial sets arising from simplicial differential graded algebras over a ring $R$.

9.1. Cohomology theories. Denote by $\Delta^{o}$ Sets the category of simplicial sets. If $A_{\bullet}^{*}$ is a simplicial differential graded $\mathbb{Z}$-algebra, then to any simplicial set $X$ we can associate a differential graded algebra $A^{*}(X)$ by taking morphisms, in $\Delta^{o}$ Sets, from $X$ to $A_{\bullet}^{*}$. In order to obtain a good cohomology theory we impose some conditions on $A_{\bullet}^{*}$, following Cartan (see [C]).

9.1.1. Let $R$ be a ring and $A_{\bullet}^{*}$ a simplicial differential graded $R$-algebra. We will assume that $A_{\bullet}^{*}$ satisfies the following axioms:

(a) Homology axiom. For each $p \geq 0$, the natural morphism $R \longrightarrow A_{p}^{*}$ is a homotopy equivalence of cochain complexes. In particular, it is a quasi-isomorphism.

(b) Homotopy axiom. For each $q \geq 0$, the simplicial set $A_{\bullet}^{q}$ is (simplicially) contractible, i.e. the homotopy groups of $A_{\bullet}^{q}$ are zero.

(c) Freeness axiom. $R$ is a principal ideal domain (PID) and, for all $p, q$, the $R$-module $A_{p}^{q}$ is free.

9.1.2. Associated to $A_{\bullet}^{*}$ there is a cohomology theory defined by

$$
A^{*}(X)=\operatorname{Hom}_{\Delta}\left(X_{\bullet}, A_{\bullet}^{*}\right),
$$

where $\operatorname{Hom}_{\Delta}$ stands for the homomorphism set in the simplicial category. $A^{*}(X)$ is a cochain complex, which in degree $q$ is equal to $\Delta^{o} \operatorname{Sets}\left(X_{\bullet}, A_{\bullet}^{q}\right)$.

Example 9.1.1. Given a ring $R$, the singular cochain complex $S^{*}(; R)$ is an example of cohomology theory in the sense above. To see this it suffices to take the simplicial $R$-algebra $S_{\bullet}^{*}(R)$ which in simplicial degree $p$ is the cochain $R$-algebra of the simplicial set represented by $p, \Delta[p]$, with evident face and degeneracies.

Cartan proves in [C], theorem 1 (see also [Maj]), that the cohomology of cohomology theories associated with simplicial differential graded algebras $A_{\bullet}^{*}$, which satisfies the homology and homotopy axioms, are isomorphic, and that this isomorphism comes from a true morphism of complexes if $A_{\bullet}^{*}$ satisfies the normalization $A_{p}^{q}=0$ if $q>p$, (loc. cit. theorem 2). Moreover, it is compatible with products if the cohomology theories satisfy some flatness conditions (loc. cit. theorem 3). We will apply 8.2.1 to obtain a comparison result at the chain level, which is stronger than Cartan's theorems.

Note that in Man, M.Mandell obtains uniqueness results for cochain theories that satisfy a different set of axioms related to the classical Eilenberg-Steenrod axioms. 
9.1.3. The category of simplicial sets $\Delta^{o}$ Sets is a monoidal category under the cartesian product. Using the algebra structure of $A_{\bullet}^{*}$ it follows that the cohomology theory $A^{*}$ defines a contravariant monoidal functor

$$
A^{*}:\left(\Delta^{o} \text { Sets }\right)^{o} \longrightarrow \mathbf{C}^{*}(R) .
$$

Theorem 9.1.2. Let $A_{\bullet}^{*}, B_{\bullet}^{*}$ be simplicial differential graded $R$-algebras satisfying axioms (a)(c). Then the contravariant monoidal functors

$$
A^{*}, B^{*}:\left(\Delta^{o} \text { Sets }\right)^{o} \longrightarrow \mathbf{C}^{*}(R),
$$

are weakly quasi-isomorphic in the category $\operatorname{Mon}\left(\left(\Delta^{o} \text { Sets }\right)^{o}, \mathbf{C}^{*}(R)\right)$. In particular, any such cohomology theory is weakly quasi-isomorphic to the singular cochain complex functor $S^{*}(; R)$.

Proof. Let $\mathbf{G}$ be the cotriple in $\Delta^{o}$ Sets which is defined, as in the topological case, by

$$
G(X)=\sqcup_{\alpha}(\Delta[\underline{n}], \alpha),
$$

where $\Delta[\underline{n}]=\Delta\left[n_{1}\right] \times \cdots \times \Delta\left[n_{r}\right]$. G induces a triple $\mathbf{T}=(T, \eta, \mu)$ in $\mathbf{M o n}\left(\Delta^{o} \mathbf{S e t s}^{o}, \mathbf{C}^{*}(R)\right)$.

By hypothesis, $H^{0}\left(A^{*}(X)\right)$ and $H^{0}\left(B^{*}(X)\right)$ are isomorphic to $H^{0}(X)$, so in order to apply the acyclic models theorem 8.2.1 we have to prove that any cohomology theory $A^{*}$ that satisfies axioms (a)-(c) is $\mathbf{T}$-acyclic and $\mathbf{T}$-presentable, the case of $S^{*}(; R)$ being well known. The $\mathbf{T}$ acyclicity will follow from the acyclicity of $A_{\bullet}^{*}$ with respect to the differential degree, while the T-presentability will follow from the contractibility with respect to the simplicial degree.

Let us first prove that $A^{*}$ is $\mathbf{T}$-acyclic. We have to prove that for any sequence $\underline{n}=\left(n_{1}, \ldots, n_{r}\right)$ the complex

$$
\ldots \longrightarrow A^{q}\left(\Delta^{\underline{n}}\right) \longrightarrow A^{q-1}\left(\Delta^{\underline{n}}\right) \longrightarrow \ldots \longrightarrow A^{0}\left(\Delta^{\underline{n}}\right) \longrightarrow H^{0}\left(A^{*}\left(\Delta^{\underline{n}}\right)\right) \longrightarrow 0,
$$

is acyclic. We prove this statement for any contractible simplicial set $X, X=\Delta^{n}$ being a special case.

Note that the $q$-cocycles of $A^{*}(X)$ are equal to $\operatorname{Hom}\left(X, Z^{q} A\right)$, where $Z^{q} A$ denotes the simplicial group of $q$-cocycles of $A_{\bullet}^{*}$. By the homology axiom $d: A_{\bullet}^{q-1} \longrightarrow Z^{q} A$ is surjective, hence it is a Kan fibration with fiber $Z^{q-1} A$, and it follows also that $Z^{q} A$ is connected.

Take a $q$-cocycle $f: X \longrightarrow Z^{q} A$. As $X$ is contractible, $f$ is homotopic to zero and $Z^{q} A$ is connected, $f$ admits an extension to a morphism $X \longrightarrow A_{\bullet}^{q-1}$, and consequently it is a boundary.

Let us now turn to $\mathbf{T}$-presentability. By the contravariant version of proposition 4.2.3, if each $A^{q}, q \geq 0$, is $\mathbf{T}$-split then $A^{*}$ will be $\mathbf{T}$-presentable. Therefore we want to define, for each $q$, a natural transformation $\theta: T\left(A^{q}\right)=A^{q} G \Rightarrow A^{q}$ such that $\theta \eta=\mathrm{id}$, where for a simplicial set $X$,

$$
\eta_{X}: A^{q}(X) \longrightarrow A^{q} G(X)=\prod_{\underline{n}} \prod_{\alpha: \Delta \underline{n} \rightarrow X} A^{q}\left(\Delta^{\underline{n}}, \alpha\right)
$$

is the morphism given by composition: $\eta_{X}(w)=(w \circ \alpha, \alpha)$. 
We denote by $X(p)$ the $p$-skeleton of the simplicial set $X$, so $X=\underline{\lim }(X(p))$. First of all, note that the functors $A^{q}$ and $A^{q} G$ are compatible with the skeleton decomposition, that is, we have

$$
\begin{aligned}
A^{q}(X) & =\lim A^{q}(X(p)), \\
A^{q} G(X) & =\underset{\lim }{ } A^{q} G(X(p)) .
\end{aligned}
$$

The first isomorphism results from the compatibility of Hom functors with limits, while for the second we observe that $G$ commutes with filtered colimits.

Thus, to define $\theta$ it is sufficient to define morphisms $\theta_{p}: A^{q} G(X(p)) \longrightarrow A^{q}(X(p)), p \geq 0$, which are sections of $\eta_{X(p)}$, and such that the diagrams

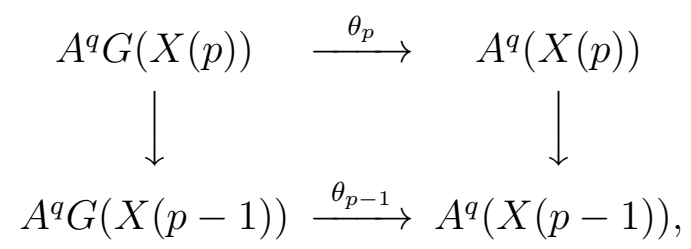

where the vertical morphisms are induced by the inclusion $X(p-1) \subseteq X(p)$, are commutative.

We will define $\theta_{p}$ inductively on $p$. The inductive step will be based on the following auxiliary result:

Extension lemma. For any $p$, the inclusion $\partial \Delta[p] \longrightarrow \Delta[p]$ induces a surjection $f_{p}$ : $A^{q}(\Delta[p]) \longrightarrow A^{q}(\partial \Delta[p])$ which has a R-linear section $s: A^{q}(\partial \Delta[p]) \longrightarrow A^{q}(\Delta[p])$.

In fact, note that, as a consequence of the homotopy axiom, for any simplicial subset $Y \subseteq X$ the induced morphism $A^{q}(X) \longrightarrow A^{q}(Y)$ is surjective, since $A_{*}^{q}$ is a contractible Kan complex and, as the inclusion $Y \subseteq X$ is a cofibration, any map $Y \longrightarrow A_{*}^{q}$ extends to a map $X \longrightarrow A_{*}^{q}$, (see [BG], and [FHT] for a more elementary proof).

In particular, the morphism $f_{p}: A^{q}(\Delta[p]) \longrightarrow A^{q}(\partial \Delta[p])$ is surjective. But $A^{q}(\partial \Delta[p])$ is $R$ projective, because $R$ is a PID and $A^{q}(\partial \Delta[p])$ is a submodule of the free $R$-module $\left(A_{p-1}^{q}\right)^{p+1}$. Thus, $f_{p}$ has an $R$-linear section $s: A^{q}(\partial \Delta[p]) \longrightarrow A^{q}(\Delta[p])$.

Let us now return to the inductive definition of $\theta_{p}$. For $p=-1$ there is nothing to prove, so let us assume that $\theta_{p-1}$ has been constructed. The $p$-skeleton $X(p)$ is obtained from the $(p-1)$-skeleton $X(p-1)$ by the pushout diagram

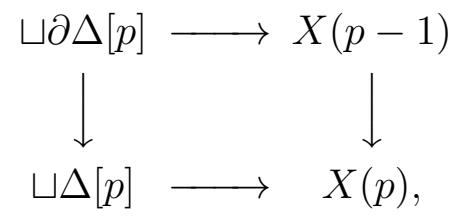

where the disjoint union is over all nondegenerated maps $\Delta[p] \longrightarrow X(p)$. Since $A^{q}$ transforms pushouts to pullbacks, we get the pullback diagram

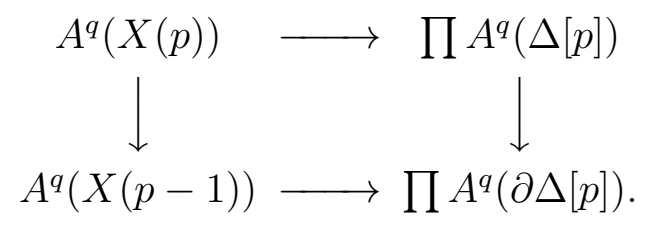


Analogously, by applying $A^{q} G$ we obtain the pullback diagram

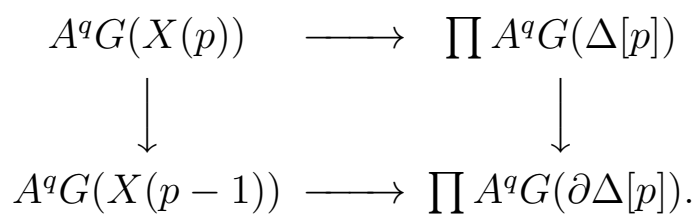

By induction we have morphisms $\theta_{p-1}=\theta_{X(p-1)}$ and $\theta_{p-1}^{\prime}=\theta_{\partial \Delta[p]}$, so it suffices to define $\theta_{p}^{\prime}=\theta_{\Delta[p]}$ to make the diagram

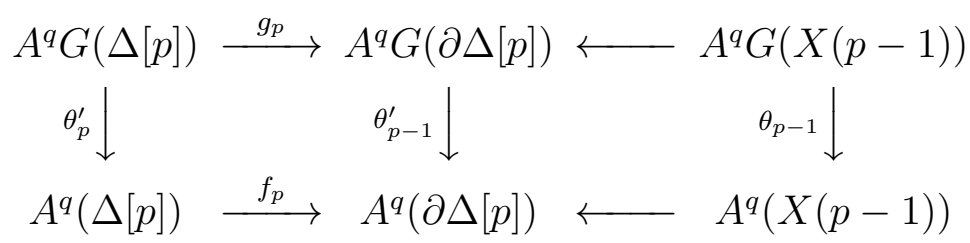

commutative and which is a section of $\eta_{\Delta[p]}$, (the square on the right commutes by induction).

Let $\pi: A^{q} G(\Delta[p]) \longrightarrow A^{q}(\Delta[p])$ be the natural projection morphism, so that $\pi \eta=\mathrm{id}$, and let $s$ be a section of $f_{p}, f_{p} s=$ id, whose existence is guaranteed by the extension lemma above. We define the morphism

$$
\theta_{p}^{\prime}=\pi+s\left(\theta_{p-1}^{\prime} g_{p}-f_{p} \pi\right)
$$

Commutativity easily follows, since

$$
f_{p} \theta_{p}^{\prime}=f_{p} \pi+f_{p} s\left(\theta_{p-1}^{\prime} g_{p}-f_{p} \pi\right)=f_{p} \pi+\theta_{p-1}^{\prime} g_{p}-f_{p} \pi=\theta_{p-1}^{\prime} g_{p} .
$$

Moreover, $\theta_{p}^{\prime}$ is a section of $\eta_{\Delta[p]}$, since

$$
\theta_{p}^{\prime} \eta_{\Delta[p]}=\pi \eta_{\Delta[p]}+s \theta_{p-1}^{\prime} g_{p} \eta_{\Delta[p]}-s f_{p} \pi \eta_{\Delta[p]}=\mathrm{id}+s f_{p}-s f_{p}=\mathrm{id},
$$

where we have used the commutativity proved above.

9.2. Any contravariant monoidal functor $F^{*}:\left(\Delta^{o} \text { Sets }\right)^{o} \longrightarrow \mathbf{C}^{*}(R)$ gives rise to a functor on $\Delta^{o}$ Sets with values in the category of $R$-differential graded algebras by composing the Künneth morphism of $F^{*}$ with the morphism induced by the diagonal of the space

$$
F^{*}(X) \otimes F^{*}(X) \stackrel{\kappa}{\longrightarrow} F^{*}(X \times X) \stackrel{\Delta^{*}}{\longrightarrow} F^{*}(X) .
$$

Now, from theorem 9.1 .2 we deduce

Corollary 9.2.1. Let $A_{\bullet}^{*}$ be a simplicial $R$-dg algebra that satisfies axioms (a)-(c). For any simplicial set $X$, the algebras $S^{*}(X ; R)$ and $A^{*}(X)$ are weakly equivalent in the category of $R$-differential graded algebras.

Example 9.2.2. As application we obtain a proof, for any simplicial set $X$, of the equivalence of the cochain algebra $S^{*}(X ; \mathbb{Q})$ and the polynomial De Rham algebra (compare with [S] and [BG]). For this, take $L_{\bullet}^{*}$ the simplicial cochain complex defined by the regular differential forms on the $\mathbb{Q}$-cosimplicial scheme $H^{\bullet}$, whose $p$ component is the hyperplane $H^{p}$ of $\mathbb{A}^{p+1}$ defined by the equation $t_{0}+\cdots+t_{p}=1$. It is a simplicial $\mathbb{Q}$-dga that satisfies the homology and homotopy axioms (see $[\mathrm{BG}]$ ), so if we define the algebra of polynomial De Rham forms of $X$ as

$$
S u^{*}(X)=\operatorname{Hom}_{\Delta}\left(X_{\bullet}, L_{\bullet}^{*}(\mathbb{Q})\right),
$$


the last corollary ensures that $S^{*}(X ; \mathbb{Q})$ and $S u^{*}(X)$ are weakly equivalent $\mathbb{Q}$-differential graded algebras. The rational field $\mathbb{Q}$ may be replaced by any field $\mathbf{k}$ of characteristic zero.

\section{REFERENCES}

[B95] M.Barr, Oriented singular homology. Theory and Applications of Categories 1, (1995), 1-9.

[B96] M.Barr, Acyclic models. Canadian Journal of Mathematics 48, (1996), 258-273.

[B02] M.Barr, Acyclic models. CRM Monograph Series, vol. 17. American Mathematical Society, 2002.

[BB] M.Barr, J.Beck, Acyclic models and triples, S. Eilenberg et alt. Proc. Conference in Categorical Algebra, La Jolla, 1965. Springer Verlag, (1966), 336-343.

[BG] A.K. Bousfield, V.K.A.M. Gugenheim, On PL De Rham theorem and rational homotopy type. Memoirs of the AMS, 179. American Mathematical Society, 1976.

[C] H. Cartan, Théories cohomologiques. Inventiones math. 35, (1976), 261-271.

[D] A. Dold, Lectures on Algebraic Topology. Springer Verlag, 1972.

[DMO] A. Dold, S. MacLane, U. Olbers, Projective classes and acyclic models, J. Bénabou et alt. Reports of te Midwest Category Seminar. Lecture Notes in Math. 47, (1967), 78-91.

[EK] S. Eilenberg, G.M.Kelly, Closed categories, S. Eilenberg et alt. Proc. Conference in Categorical Algebra, La Jolla, 1965. Springer Verlag, (1966), 421-562.

[EM1] S. Eilenberg, S.McLane, Acyclic models, American Journal of Mathematics 75, (1953), 189-199.

[EM2] S. Eilenberg, S.McLane, On the groups $H(\pi, n)$, I. Annals of Math. 58, (1953), 55-106.

[FHT] Y. Félix, S. Halperin, J.C. Thomas, Rational homotopy theory. Springer Verlag, 2001.

[GK] E. Getzler, M. Kapranov, Modular operads, Compositio Math. 110 (1998), 65-126.

[GM] V.K.A.M Gugenhein, J.P. May, On the theory and applications of differential torsion products. Memoirs of the AMS, 142. American Mathematical Society, 1974.

[GMo] V.K.A.M. Gugenheim, J.C. Moore, Acyclic models and fibre spaces. TAMS 85, (1957), 265-306.

[GN] F. Guillén, V. Navarro Aznar, Un critère d'extension des foncteurs définis sur les schémas lisses. Publ. Math. I.H.E.S. 95 (2002), 1-91.

[GNPR] F. Guillén Santos, V. Navarro, P. Pascual, A. Roig, Moduli spaces and formal operads, alg-geom/9402098, February 2004. Duke Math. Journal, to appear.

[GZ] P. Gabriel, M. Zisman, Calculus of fractions and homotopy theory. Springer Verlag, 1967.

[K1] H. Kleisli, Resolutions in additive and non-additive categories. Queen's Papers in Pure and Applied Mathematics 32, 1973.

[K2] H. Kleisli, On the construction of standard complexes. Journal of Pure and Appl. Algebra 4, (1974), $243-260$.

[KS] G. M. Kelly, R. Street, Review of elements of 2-categories. Springer Lecture Notes in Math. 420, (1974), 75-103.

[Ko] M. Kontsevich, Operads and motives in deformation quantization, Lett. Math. Physics 48 (1999), 35-72.

[ML] S. MacLane, Categories for the working mathematician. Graduate Text in Mathematics, 5. Springer Verlag, 1971.

[Maj] M. Majewski, Rational homotopical models and uniqueness. Memoirs of the AMS, 143. American Mathematical Society, 2000.

[Man] M.A. Mandell, Cochain multiplications. American Journal of Math. 124, (2002), 547-566.

[MSS] M. Markl, S. Schneider, J. Stasheff, Operads in Algebra, Topology and Physics. Surveys and Monographs, 96. American Mathematical Society, 2002.

[Mas] W. Massey, Singular homology theory. Springer GTM 70, 1980.

[Mu] H.J. Munkholm, Shm maps of differential graded algebras. Journal of Pure and Appl. Algebra 13, (1978), $221-232$.

[S] R. G. Swan, Thom's theory of differential forms on simplicial sets. Topology, 14, (1975), 271-273.

[T] D. Tamarkin, Formality of chain operad of little discs. Letters in Math. Physics 66, (2003), 65-72.

(F. Guillén Santos and V. Navarro) Departament D’Àlgebra i Geometria, Universitat de Barcelona, Gran Via 585, 08007 BARCELONA (SPAin)

(P. Pascual and A. Roig) Departament de Matemàtica Aplicada I, Universitat Politècnica de Catalunya, Diagonal 647, 08028 Barcelona (Spain).

E-mail address: fguillen@ub.edu, pere.pascual@upc.edu, agustin.roig@upc.edu 